\title{
Article \\ Multi-Omics Approach to Elucidate Cerebrospinal Fluid Changes in Dogs with Intervertebral Disc Herniation
}

\author{
Anita Horvatić $1, *\left(\right.$ ) Andrea Gelemanović ${ }^{2}$, , Boris Pirkić $^{3}$, Ozren Smolec ${ }^{3, *}$, Blanka Beer Ljubić ${ }^{4}$, Ivana Rubić ${ }^{4}$, \\ Peter David Eckersall ${ }^{5}{ }^{\circ}$, Vladimir Mrljak ${ }^{4}{ }^{-}$, Mark McLaughlin ${ }^{5}$, Marko Samardžija ${ }^{6}$ and Marija Lipar ${ }^{3}$ \\ 1 Department of Chemistry and Biochemistry, Faculty of Food Technology and Biotechnology, \\ University of Zagreb, 10000 Zagreb, Croatia \\ 2 Mediterranean Institute for Life Sciences, 21000 Split, Croatia; andrea.gelemanovic@gmail.com \\ 3 Clinic for Surgery, Orthopaedics and Ophthalmology, Faculty of Veterinary Medicine, University of Zagreb, \\ 10000 Zagreb, Croatia; bpirkic@vef.hr (B.P.); mlipar@vef.hr (M.L.) \\ 4 Internal Diseases Clinic, Faculty of Veterinary Medicine, University of Zagreb, 10000 Zagreb, Croatia; \\ bla.ljubic@gmail.com (B.B.L.); irubic@vef.hr (I.R.); vmrljak@vef.hr (V.M.) \\ 5 Institute of Biodiversity, Animal Health \& Comparative Medicine, College of Medicine, \\ Veterinary Medicine and Life Sciences, University of Glasgow, Glasgow G12 8QQ, UK; \\ David.Eckersall@glasgow.ac.uk (P.D.E.); mark.mclaughlin@glasgow.ac.uk (M.M.) \\ 6 Reproduction and Obstetrics Clinic, Faculty of Veterinary Medicine, University of Zagreb, \\ 10000 Zagreb, Croatia; smarko@vef.hr \\ * Correspondence: ahorvatic@pbf.hr (A.H.); osmolec@vef.hr (O.S.)
}

\section{check for} updates

Citation: Horvatić, A.; Gelemanović, A.; Pirkić, B.; Smolec, O.; Beer Ljubić, B.; Rubić, I.; Eckersall, P.D.; Mrljak, V.; McLaughlin, M.; Samardžija, M.; et al. Multi-Omics Approach to Elucidate Cerebrospinal Fluid Changes in Dogs with Intervertebral Disc Herniation. Int. J. Mol. Sci. 2021, 22, 11678. https://doi.org/10.3390/ijms222111678

Academic Editors: Federica Iavarone and Andrea Urbani

Received: 22 September 2021

Accepted: 25 October 2021

Published: 28 October 202

Publisher's Note: MDPI stays neutral with regard to jurisdictional claims in published maps and institutional affiliations.

Copyright: (c) 2021 by the authors. Licensee MDPI, Basel, Switzerland. This article is an open access article distributed under the terms and conditions of the Creative Commons Attribution (CC BY) license (https:// creativecommons.org/licenses/by/ $4.0 /)$.
Abstract: Herniation of the intervertebral disc (IVDH) is the most common cause of neurological and intervertebral disc degeneration-related diseases. Since the disc starts to degenerate before it can be observed by currently available diagnostic methods, there is an urgent need for novel diagnostic approaches. To identify molecular networks and pathways which may play important roles in intervertebral disc herniation, as well as to reveal the potential features which could be useful for monitoring disease progression and prognosis, multi-omics profiling, including highresolution liquid chromatography-mass spectrometry (LC-MS)-based metabolomics and tandem mass tag (TMT)-based proteomics was performed. Cerebrospinal fluid of nine dogs with IVDH and six healthy controls were used for the analyses, and an additional five IVDH samples were used for proteomic data validation. Furthermore, multi-omics data were integrated to decipher a complex interaction between individual omics layers, leading to an improved prediction model. Together with metabolic pathways related to amino acids and lipid metabolism and coagulation cascades, our integromics prediction model identified the key features in IVDH, namely the proteins follistatin Like 1 (FSTL1), secretogranin V (SCG5), nucleobindin 1 (NUCB1), calcitonin re-ceptorstimulating peptide 2 precursor (CRSP2) and the metabolites $\mathrm{N}$-acetyl-D-glucosamine and adenine, involved in neuropathic pain, myelination, and neurotransmission and inflammatory response, respectively. Their clinical application is to be further investigated. The utilization of a novel integrative interdisciplinary approach may provide new opportunities to apply innovative diagnostic and monitoring methods as well as improve treatment strategies and personalized care for patients with degenerative spinal disorders.

Keywords: cerebrospinal fluid; dog; integromics; intervertebral disc herniation; metabolomics; proteomics; personalized medicine

\section{Introduction}

Lower back pain (LBP) is a common disorder with a lifetime prevalence over $70 \%$ in the global human population, causing major human health problems and extensive health care utilization [1]. LBP may be caused by degenerative spinal disorders (intervertebral disc damage- - herniation or fractures), vertebral infections, or even cancer [2]. Although experimental animals have been used to study degenerative spinal disorders, according 
to $3 \mathrm{R}$ (reduction, refinement and replacement) principles, dogs are reported as the most suitable translational model for biomechanical studies and surgical procedures of the spine and related pathophysiological processes $[1,3,4]$. In dogs, intervertebral disc herniation (IVDH)-related diseases are the most common cause of spinal cord injury and sensory deficiency [1]. The canine intervertebral disc (IVD) is an elastic structure located between the bony vertebrae and is responsible for the stability and flexibility of the vertebral column [5]. The healthy intervertebral disc consists of the inner nucleus pulposus (NP) encompassing the annulus fibrosus (AF) which, together with the cartilaginous endplates, abut the vertebrae [6]. The nucleus is rich with proteoglycan, an incompressible shock absorber which under compressive loads, distributes hydraulic pressure in all directions $[3,6]$, while the AF consists of collagen type I, elastin and fibroblast-like cells. The IVD is aneural and avascular, receiving nutrition from adjacent cartilaginous endplates [3]. However, due to aging, as well as various environmental factors (e.g., mechanical stress and osmotic pressure), the IVD is susceptible to mechanical and chemical degeneration. At the molecular level, the IVDH is mostly related to the extracellular matrix molecules and collagen degradation, resulting in the upregulation of their degradation products as well as the upregulation of pro-inflammatory cytokines $[7,8]$. Once the degenerative process starts, a biochemical and mechanical cascade of events is triggered that can ultimately lead to structural failure of the IVD and clinical signs of diseases [5,9].

IVDH is a common cause of spinal cord injury (SCI) in dogs, resulting in the compression of the spinal cord, associated with pain as well as sensory and motor deficits. The severity of neurological signs is determined by neuroanatomical location, velocity and the amount of the compressive material as well as the duration of the compression [10]. Primary SCI refers to the initial mechanical insult, whereas secondary injury is a biochemical cascade following the primary event and consists of vascular dysregulation, neurogenic shock, oxidative stress and excitotoxicity, ischemia and inflammatory cascade [4]. Nevertheless, the IVDH-related pathophysiological processes remain poorly understood, especially at the molecular level. Diagnosis at an early stage of the disease could help to restore disc structure and mechanical structure by using personalized and targeted therapeutic strategies, circumventing the painful symptoms in patients [11,12].

The use of state-of-the-art technology and instrumentation has enabled the identification and quantification of metabolites and large biomolecules in complex matrices with high precision, reproducibility and sensitivity, using low analyte concentrations. Shotgun tandem mass tag (TMT)-based quantitative proteomics enables high-throughput multiplex analysis on up to sixteen samples simultaneously. Unlike proteomics, metabolomics is a powerful tool to reveal metabolomic panels since metabolites are common intermediate and end products of complex biochemical cascades linking the genome, transcriptome and proteome to the phenotype [13]. High-resolution mass spectrometry-based proteomics and metabolomics, combined with the application of various bioinformatic tools and pipelines, have been widely used for studying the pathophysiological processes in central nervous system (CNS) diseases and other neurological conditions by analyzing tissues and biological fluids such as serum or cerebrospinal fluid (CSF) [13-15]. Although not easily accessible, unlike blood, due to its multiple functions such as structural, hydrodynamic, metabolic and immunological, CSF is the only readily accessible biofluid that evaluates current CNS status and is a key consideration in differential diagnostic procedures in neurodegenerative disorders [14-16]. The major constituent of cerebrospinal fluid is water $(99 \%)$, whereas the remaining $1 \%$ are ions, proteins, glucose, lactose and other organic compounds and cells. The chemical composition of CSF is affected by CNS-related pathophysiological processes [15].

Both human and animal clinical studies are mainly focused on a specific omics layer rather than applying a multi-omics approach, which is the main reason why full omics integration is still lacking, especially those providing statistical methods for consistent multi-omics data handling [16]. However, the number of integromics-related studies is constantly increasing. The integration of multi-omics data through data- and knowledge- 
based approaches enables insight into complex events occurring at the molecular level that are being reflected in various pathophysiological processes in organisms and that cannot be observed by studying just one component of a biological system. Finally, the major challenge in multiple omics data integration lies in the selection of appropriate tools for a given research question [17].

In our study, integrative MS-based high-resolution proteomic and untargeted metabolomics analyses of CSF have been employed to gain deeper insights into changes occurring during IVDH in dogs, monitoring the pathophysiological processes at the protein and metabolite levels. Furthermore, since there is no "golden standard" for integrating proteomic and metabolomics data, we designed a classification model to identify the most important features and, by using data-mining tools, reveal significant pathways affected by this degenerative disease. Special focus was placed on identifying the most consistent features being the revelation of potential biomarkers of spinal cord compression, as well as the effect of changes in CSF on CNS and vital functions. The understanding of the patient's unique profiles at the molecular level and pathways affected could help to improve the diagnosis of this disease and select specific treatments to provide the best outcome.

\section{Results}

\subsection{Proteomic Analysis and Proteomic Data Validation}

Proteomic analysis of CSF was performed using a gel-free tandem mass tag labelbased proteomic approach without the depletion of high abundant proteins. Prior to the analysis, total protein concentration in CSF was measured. However, there were no significant differences in total protein concentration between the control and IVDH groups (data not shown). Data-dependent analysis (DDA) enabled the identification of 626 CSF proteins according to set criteria (two unique peptides, $5 \%$ false discovery rate, FDR), among which 204 proteins were master proteins. The information about peptide spectrum matches (PSMs), including identified peptides, proteins and protein groups is available in Supplementary File S1. The principal component analysis (PCA) score plot revealed two clear clusters of samples, with the IVDH group being more heterogeneous based on TMT-based proteomic results (Supplementary Figure S1).

In total, 227 proteins were statistically significantly differential in abundance due to the disc herniation after applying FDR correction (Supplementary File S2, Figure 1a,b), with a majority of proteins ( $89 \%$ ) being down-regulated in the IVDH group compared to the control group. Of those, 73 proteins were determined as being related to unique genes (Supplementary Table S1) with the ten most upregulated and downregulated proteins shown in Table 1.

Table 1. The top 10 most significantly changed proteins in cerebrospinal fluid of dogs with intervertebral disc herniation (IVDH) compared to healthy dogs. Complete list of all significantly changed proteins can be found in Supplementary File S2.

\begin{tabular}{|c|c|c|c|c|c|c|c|}
\hline \multicolumn{4}{|c|}{$\begin{array}{c}\text { Higher in Abundance in IVDH Group } \\
\text { (Upregulated) }\end{array}$} & \multicolumn{4}{|c|}{$\begin{array}{c}\text { Higher in Abundance in Control Group } \\
\text { (Downregulated) }\end{array}$} \\
\hline $\begin{array}{l}\text { Accession } \\
\text { No. (NCBI) }\end{array}$ & $\begin{array}{l}\text { Adjusted } \\
p \text {-Value }\end{array}$ & $\log 2 \mathrm{FC}$ & $\begin{array}{l}\text { Gene } \\
\text { Symbol }\end{array}$ & $\begin{array}{l}\text { Accession } \\
\text { No. (NCBI) }\end{array}$ & $\begin{array}{l}\text { Adjusted } \\
p \text {-Value }\end{array}$ & $\log 2 \mathrm{FC}$ & $\begin{array}{l}\text { Gene } \\
\text { Symbol }\end{array}$ \\
\hline 359321961 & 0.0147 & 0.31 & $\mathrm{~F} 2$ & 345789648 & 0.0056 & -2.00 & CHGB \\
\hline 73975389 & 0.0349 & 0.37 & AFM & 1418342647 & 0.0075 & -1.82 & SCG2 \\
\hline 1239925760 & 0.0181 & 0.39 & C5 & 345795204 & 0.0056 & -1.81 & SCG5 \\
\hline 18139619 & 0.0181 & 0.41 & PLG & 3046976 & 0.0266 & -1.58 & COL1A1 \\
\hline 1418515495 & 0.0181 & 0.43 & AHSG & 1239931864 & 0.0056 & -1.43 & ENPP2 \\
\hline 2147092 & 0.0181 & 0.48 & ALB & 73970011 & 0.0181 & -1.38 & $\mathrm{C} 10 \mathrm{H} 2$ orf 40 \\
\hline 3915607 & 0.0266 & 0.50 & APOA1 & 1418314244 & 0.0098 & -1.31 & TTR \\
\hline 1239918269 & 0.0056 & 0.52 & AOC3 & 1239976444 & 0.0056 & -1.30 & FSTL1 \\
\hline 73975797 & 0.0098 & 0.68 & PON1 & 1418222978 & 0.0056 & -1.30 & CRSP2 \\
\hline 1418304654 & 0.0349 & 1.01 & APOC1 & 197085524 & 0.0056 & -1.30 & CRSP4 \\
\hline
\end{tabular}



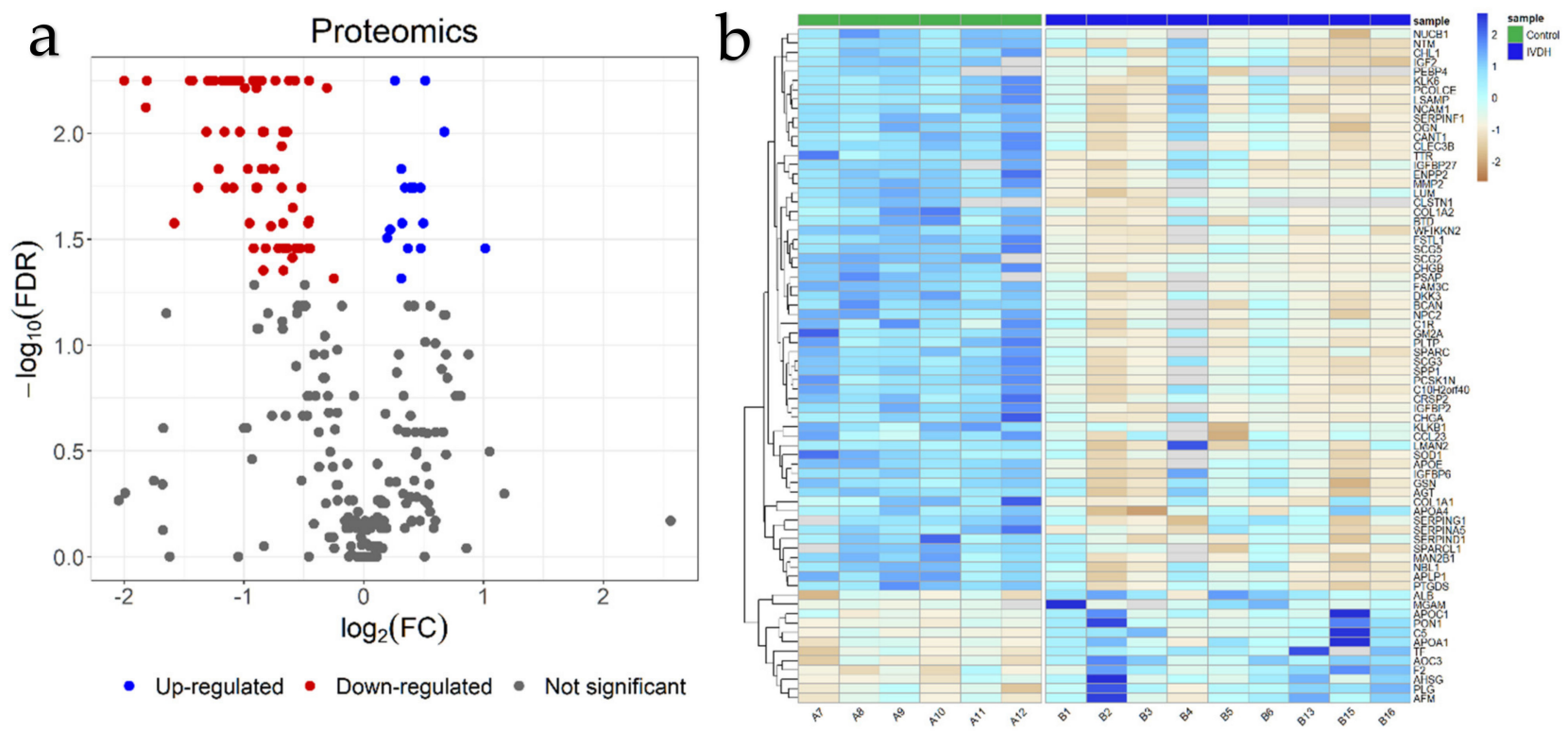

Figure 1. Statistical analysis of proteomics results: (a) Volcano plot of evaluated groups after false discovery rate (FDR) correction. Proteins overexpressed in intervertebral disc herniation (IVDH) group are in red, lower-expressed are in blue. (b) Heatmap revealing the differentially expressed genes in the control and intervertebral disc herniation groups. Each row represents the fold change (FC) expressed as log2FC of a single gene across all samples. Figure $1 \mathrm{~b}$ is also available within Supplementary Files for detailed insight (Supplementary Figure S9).

According to gene ontology analysis (FDR corrected $p$-values $<0.05)$ using the Database for Annotation, Visualization and Integrated Discovery (DAVID) tools, proteins with significant differences in abundances within the control and IVDH group were involved in calcium ion binding $(30.6 \%)$, serine-type endopeptidase inhibitor activity $(19.4 \%)$, heparin binding $(13.8 \%)$, cholesterol binding $(11.1 \%)$, phosphatidylcholine-sterol O-acyltransferase activator activity $(8.3 \%)$, collagen binding $(8.3 \%)$ and fatty acid binding $(8.3 \%)$, as can be found in Supplementary Figure S2 and Supplementary File S3.

REACTOME pathway analysis (Supplementary File S3; filtered list presented in Table 2) revealed that most of the CSF proteins differing in abundance are involved in the metabolism of proteins $\left(26, p=3.46 \times 10^{-8}\right)$, the immune system $\left(19, p=2.70 \times 10^{-4}\right)$, hemostasis $\left(19, p=1.14 \times 10^{-11}\right)$ and the regulation of Insulin-like Growth Factor (IGF) transport and uptake $\left(19, p=5.86 \times 10^{-23}\right)$. The major interacting proteins involved in the most significant pathways are depicted in Figure 2.

Table 2. REACTOME pathways affected by intervertebral disc herniation in canine cerebrospinal fluid (filtered list based on the relation to the disease). Complete list can be found in Supplementary File S3.

\begin{tabular}{|c|c|c|c|c|}
\hline REACTOME Pathway Name & ID & \# Genes & $\begin{array}{c}\text { \# Background } \\
\text { Genes }\end{array}$ & $\begin{array}{l}\text { Adjusted } \\
p \text {-Value }\end{array}$ \\
\hline $\begin{array}{c}\text { Regulation of Insulin-like Growth Factor (IGF) } \\
\text { transport and uptake by Insulin-like Growth Factor } \\
\text { Binding Proteins (IGFBPs) }\end{array}$ & HSA-381426 & 19 & 123 & $5.86 \times 10^{-23}$ \\
\hline Post-translational protein phosphorylation & HSA-8957275 & 13 & 106 & $2.79 \times 10^{-14}$ \\
\hline Platelet activation, signaling and aggregation & HSA-76002 & 16 & 256 & $1.04 \times 10^{-13}$ \\
\hline Hemostasis & HSA-109582 & 19 & 601 & $1.14 \times 10^{-11}$ \\
\hline Extracellular matrix organization & HSA-1474244 & 12 & 298 & $2.69 \times 10^{-8}$ \\
\hline Metabolism of proteins & HSA-392499 & 26 & 1948 & $3.46 \times 10^{-8}$ \\
\hline Binding and uptake of ligands by scavenger receptors & HSA-2173782 & 6 & 40 & $3.17 \times 10^{-7}$ \\
\hline
\end{tabular}


Table 2. Cont.

\begin{tabular}{|c|c|c|c|c|}
\hline REACTOME Pathway Name & ID & \# Genes & $\begin{array}{l}\text { \# Background } \\
\text { Genes }\end{array}$ & $\begin{array}{l}\text { Adjusted } \\
p \text {-Value }\end{array}$ \\
\hline Intrinsic pathway of fibrin clot formation & HSA-140837 & 5 & 22 & $7.83 \times 10^{-7}$ \\
\hline ECM proteoglycans & HSA-3000178 & 6 & 75 & $7.48 \times 10^{-6}$ \\
\hline Degradation of the extracellular matrix & HSA-1474228 & 7 & 139 & $1.30 \times 10^{-5}$ \\
\hline Post-translational protein modification & HSA-597592 & 17 & 1366 & $6.26 \times 10^{-5}$ \\
\hline Plasma lipoprotein remodeling & HSA-8963899 & 4 & 30 & $7.28 \times 10^{-5}$ \\
\hline Chylomicron remodeling & HSA-8963901 & 3 & 10 & $1.20 \times 10^{-4}$ \\
\hline Innate immune system & HSA-168249 & 14 & 1012 & $1.20 \times 10^{-4}$ \\
\hline Regulation of complement cascade & HSA-977606 & 4 & 47 & $2.70 \times 10^{-4}$ \\
\hline Neutrophil degranulation & HSA-6798695 & 9 & 471 & $3.70 \times 10^{-4}$ \\
\hline Crosslinking of collagen fibrils & HSA-2243919 & 3 & 18 & $3.90 \times 10^{-4}$ \\
\hline Metabolism of vitamins and cofactors & HSA-196854 & 6 & 185 & $4.20 \times 10^{-4}$ \\
\hline Amyloid fiber formation & HSA-977225 & 4 & 78 & $1.30 \times 10^{-3}$ \\
\hline Integrin cell surface interactions & HSA-216083 & 4 & 83 & $1.60 \times 10^{-3}$ \\
\hline Activation of matrix metalloproteinases & HSA-1592389 & 3 & 33 & $1.60 \times 10^{-3}$ \\
\hline $\begin{array}{c}\text { Diseases associated with glycosaminoglycan } \\
\text { metabolism }\end{array}$ & HSA-3560782 & 3 & 40 & $2.50 \times 10^{-3}$ \\
\hline Collagen degradation & HSA-1442490 & 3 & 64 & $7.20 \times 10^{-3}$ \\
\hline Glycosphingolipid metabolism & HSA-1660662 & 2 & 44 & $3.68 \times 10^{-2}$ \\
\hline Glycosaminoglycan metabolism & HSA-1630316 & 3 & 122 & $3.22 \times 10^{-2}$ \\
\hline
\end{tabular}

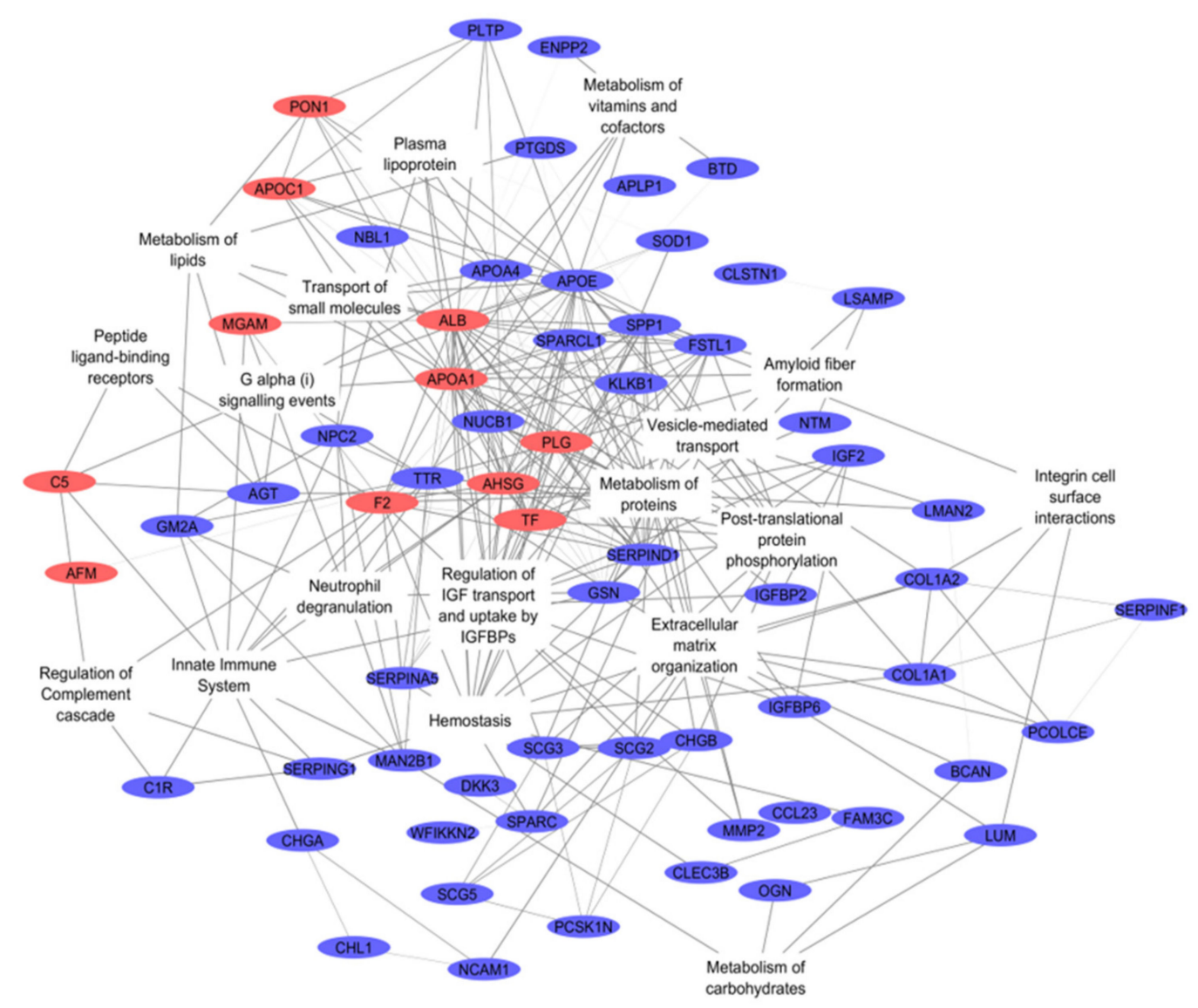

Figure 2. Interactome of pathways differentially expressed between control and intervertebral disc herniation (IVDH) groups and their intermediate proteins. The network was visualized using the Cytoscape using the results of REACTOME pathway analysis and protein-protein interactions identified with STRINGdb. Proteins overexpressed in IVDH are in red, lower-expressed are in blue. 
The differences in protein abundance revealed by MS-based proteomic analysis were evaluated by measuring the activity of paraoxonase 1 (PON1) as well as albumin concentration in CSF samples to validate the proteomic results. Significant changes in the abundance of albumin (difference between means $\pm \mathrm{SEM}=1522.0 \pm 423.4 ; p=0.0058$ ) and PON1 activity (difference between means $\pm \mathrm{SEM}=189.6 \pm 77.79 \mathrm{mU} / \mathrm{L} ; p=0.0375$ ) were found in the IVDH group (Figure 3). The obtained results for albumin and PON1 are in line with the proteomic findings; however, the validation results (if converted to fold change) have higher fold changes than those obtained by proteomic analysis, which cloud be explained by the sensitivity of TMT technology [18].

ALB

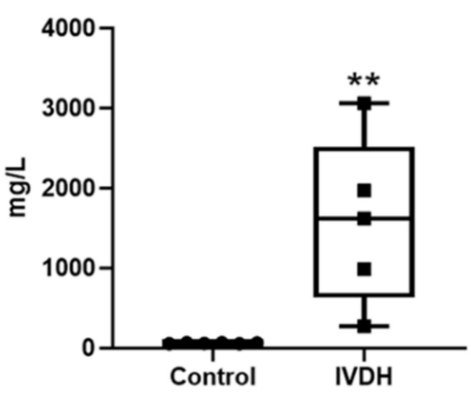

(a)

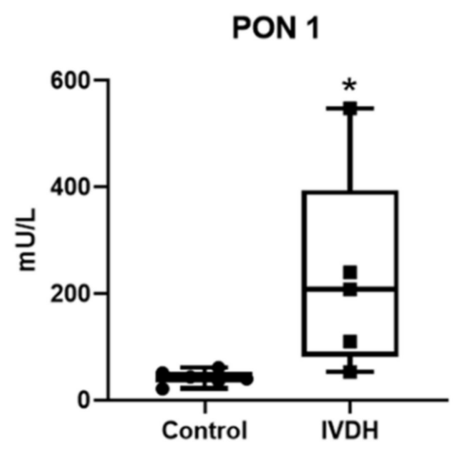

(b)

Figure 3. Analytical validation of proteomic results: (a) measured activity of paraoxonase 1 (PON1), (b) albumin (ALB) concentration in cerebrospinal fluid of intervertebral disc herniation (IVDH) group compared to the control group. ${ }^{*} p<0.05$ ( $p=0.0375$ herein), ${ }^{* *} p<0.01$ ( $p=0.0058$ herein).

\subsection{Metabolomics}

Global metabolome analysis using the zwitterionic-polymeric hydrophilic interaction chromatographic separation of metabolites in CSF resulted in 2941 peaks representing metabolic features, which were annotated by database search or identified on the basis of mass and mass/retention time match to known standards, respectively. Among them, 55 unique compounds were matched to a known standard and another 3798 potential metabolites were annotated in (Polyomics integrated Metabolomics Pipeline) PiMP, using available databases. The list of metabolites generated using PiMP software is available in Supplementary File S4. A PCA score plot revealed that the metabolomic profiles of the studied groups (healthy control and IVDH) could be clearly distinguished (Supplementary Figure S3) and 291 metabolic features referring to 73 metabolites according to PiMP analysis (Figure 4a, Supplementary File S4) were significantly changed. Additionally, 14 significantly changed metabolites were identified using standards (Table 3, Figure $4 b)$. Total ion chromatograms showing CSF metabolites for both groups in positive and negative ion mode are shown in Supplementary Figures S4-S7. 

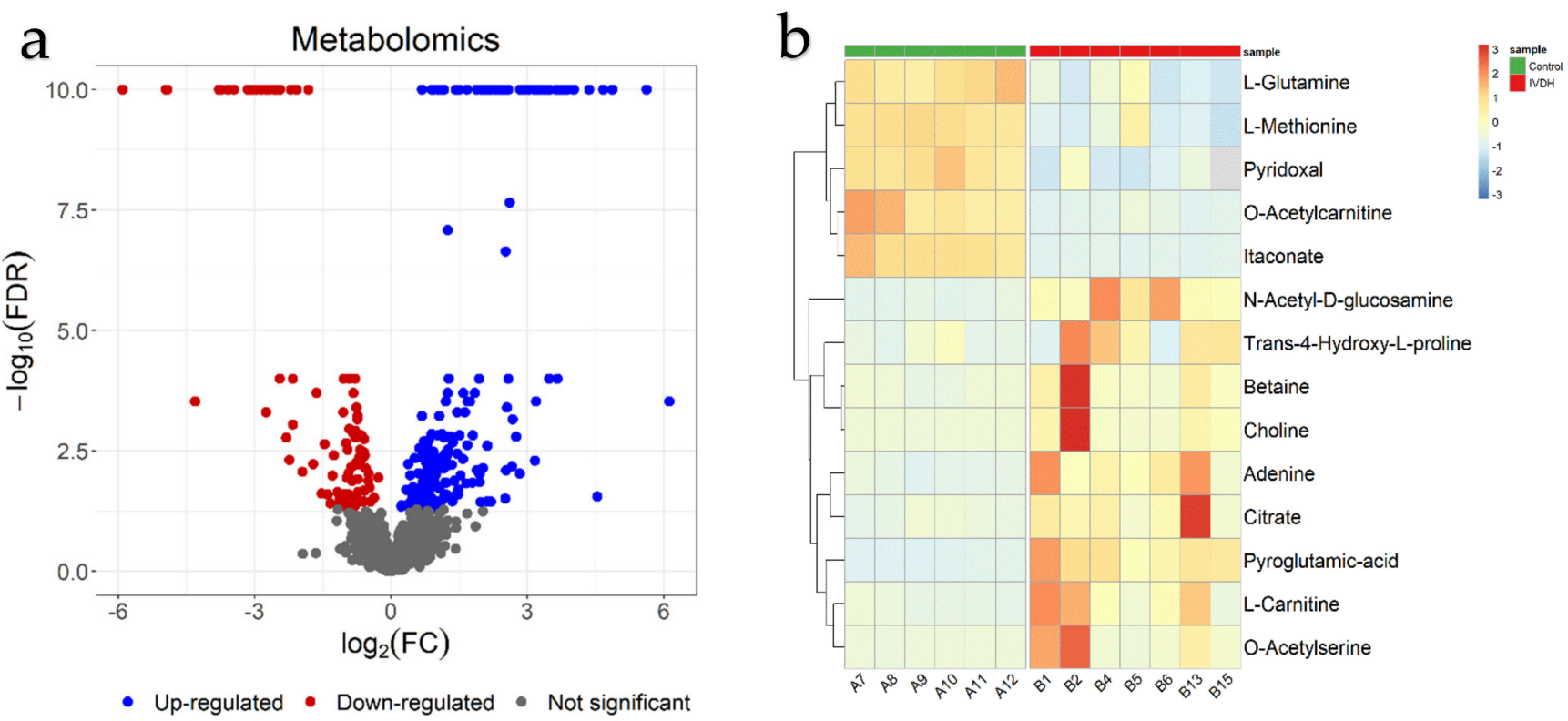

Figure 4. Statistical analysis of proteomics results: (a) Volcano plot of evaluated groups after false discovery rate (FDR) correction. Proteins overexpressed in intervertebral disc herniation (IVDH) group are in red, lower-expressed are in blue.; (b) Heatmap revealing the differentially expressed genes in the control and IVDH groups. Each row represents fold change (FC) expressed as the log2FC of a single gene across all samples.

Table 3. List of identified (using authentic standards) significantly changed metabolites in cerebrospinal fluid of intervertebral disc herniation (IVDH) group compared to healthy dogs obtained using the Polyomics integrated Metabolomics Pipeline (PiMP).

Higher in Abundance in IVDH Group (Upregulated)

Higher in Abundance in Control Group (Downregulated)

\begin{tabular}{cccccccc}
\hline Peak ID & Metabolite & $\mathbf{l o g}$ FC & $\begin{array}{c}\text { Adjusted } \\
p \text {-Value }\end{array}$ & Peak ID & Metabolite & $\begin{array}{c}\text { log2FC } \\
p \text {-Value }\end{array}$ \\
\hline 693 & Hydroxyproline & 0.76 & $3.00 \times 10^{-2}$ & 2325 & Itaconate & -3.78 & $5.1 \times 10^{-10}$ \\
23 & Betaine & 1.08 & $4.16 \times 10^{-2}$ & 858 & O-Acetylcarnitine & -2.73 & $4.6 \times 10^{-6}$ \\
1789 & Citrate & 1.19 & $1.44 \times 10^{-2}$ & 847 & Pyridoxal & -2.30 & 0.0017 \\
1410 & N-Acetyl-D- & 1.46 & $5.00 \times 10^{-4}$ & 2717 & L-Methionine & -2.16 & 0.0009 \\
688 & glucosamine & 1.51 & $1.50 \times 10^{-2}$ & 2070 & L-Glutamine & -0.83 & 0.0002 \\
217 & Adenine & 1.65 & $1.51 \times 10^{-2}$ & & & & \\
24 & L-Carnitine & Choline & 1.79 & $1.46 \times 10^{-2}$ & & & \\
466 & L-Glutamate & 2.51 & $3.10 \times 10^{-2}$ & & & \\
245 & Pyroglutamic acid & 2.52 & $2.30 \times 10^{-7}$ & & & & \\
\hline
\end{tabular}

KEGG Pathway analysis performed in PiMP revealed the CSF metabolites changed due to the IVDH are involved in C5-branched dibasic acid metabolism $\left(p=7.6 \times 10^{-9}\right)$, D-glutamine and D-glutamate metabolism $\left(p=7.5 \times 10^{-6}\right)$, and Arginine and proline metabolism $\left(p=2.8 \times 10^{-4}\right)$, among others, as reported in Table 4 . 
Table 4. Metabolic pathways affected by intervertebral disc herniation in canine cerebrospinal fluid generated by the Polyomics integrated Metabolomics Pipeline (PiMP) using KEGG Pathway.

\begin{tabular}{|c|c|c|c|c|}
\hline KEGG Pathway Name & Pathway ID & $\begin{array}{l}\text { Assigned } \\
\text { Formulas }\end{array}$ & $\begin{array}{c}\text { Total Pathway } \\
\text { Formulas }\end{array}$ & $\begin{array}{c}\text { Pathway } \\
\text { Coverage }(\%)\end{array}$ \\
\hline C5-Branched dibasic acid metabolism & 99 & 7 & 21 & 33.33 \\
\hline D-Glutamine and D-glutamate metabolism & 55 & 4 & 7 & 57.14 \\
\hline Glyoxylate and dicarboxylate metabolism & 93 & 11 & 48 & 22.92 \\
\hline Ascorbate and aldarate metabolism & 7 & 12 & 25 & 48.00 \\
\hline Arginine and proline metabolism & 35 & 25 & 79 & 31.65 \\
\hline GABAergic synapse & 218 & 3 & 9 & 33.33 \\
\hline Histidine metabolism & 37 & 21 & 41 & 51.22 \\
\hline Butanoate metabolism & 98 & 6 & 30 & 20.00 \\
\hline Pentose and glucuronate interconversions & 4 & 8 & 23 & 34.78 \\
\hline Chlorocyclohexane and chlorobenzene degradation & 41 & 3 & 57 & 5.26 \\
\hline Citrate cycle (TCA cycle) & 2 & 4 & 15 & 26.67 \\
\hline Phosphotransferase system (PTS) & 155 & 3 & 25 & 12.00 \\
\hline Cysteine and methionine metabolism & 28 & 11 & 52 & 21.15 \\
\hline Glutathione metabolism & 58 & 5 & 29 & 17.24 \\
\hline Proximal tubule bicarbonate reclamation & 243 & 5 & 16 & 31.25 \\
\hline Glutamatergic synapse & 215 & 2 & 7 & 28.57 \\
\hline Nitrogen metabolism & 124 & 2 & 17 & 11.76 \\
\hline Vitamin B6 metabolism & 106 & 4 & 29 & 13.79 \\
\hline Glycerolipid metabolism & 73 & 4 & 14 & 28.57 \\
\hline $\mathrm{ABC}$ transporters & 152 & 32 & 80 & 40.00 \\
\hline Alanine, aspartate and glutamate metabolism & 24 & 9 & 23 & 39.13 \\
\hline Purine metabolism & 20 & 10 & 78 & 12.82 \\
\hline Glycerophospholipid metabolism & 76 & 3 & 23 & 13.04 \\
\hline HIF-1 signaling pathway & 167 & 3 & 12 & 25.00 \\
\hline Zeatin biosynthesis & 122 & 4 & 30 & 13.33 \\
\hline Bile secretion & 251 & 10 & 89 & 11.24 \\
\hline Pyrimidine metabolism & 23 & 13 & 56 & 23.21 \\
\hline Galactose metabolism & 6 & 5 & 21 & 23.81 \\
\hline Mineral absorption & 253 & 13 & 26 & 50.00 \\
\hline
\end{tabular}

\subsection{Integration of Omics Data}

Subgroups of significantly differentially abundant protein and metabolite sets were subsequently analyzed by applying both data-driven and knowledge based-approaches as specified within the methods section. Firstly, in order to extract the important information from the obtained omics features, PCA analysis was performed, showing the separation of samples when plotted against the first two principal components (Supplementary Figure S8). Furthermore, we built various classification models with integrated data from 16 significant and identified metabolites and 73 significant and unique proteins. In addition, we used two different algorithms for feature selection-recursive feature elimination (RFE) and minimal-redundancy-maximal-relevance (mRMR) algorithm, and the results (in the form of confusion matrices and feature ranks based on their importance) are reported in Figure 5. The RFE algorithm showed two best models with 7 and 9 features selected, while the best performance of mRMR algorithm showed one model with the top 30 ranked features. Since these classification models are built upon a very small training set (only 9 samples), in order to have some samples left for the test set (4 samples), we built a final classification model from the features that were constantly selected, regardless of the algorithm. Thus, features appearing in 2/3 models (RFE with 7 and 9 selected features and mRMR with top 30 ranked features) were used for the final classification model. Selected features for the final classification model were: follistatin Like 1 (FSTL1), secretogranin V (SCG5), nucleobindin 1 (NUCB1), calcitonin receptor-stimulating peptide 2 precursor (CRSP2), $\mathrm{N}$-acetyl-D-glucosamine and adenine. The final model, as well as models that used the RFE algorithm, correctly classified all the samples in the test set. 


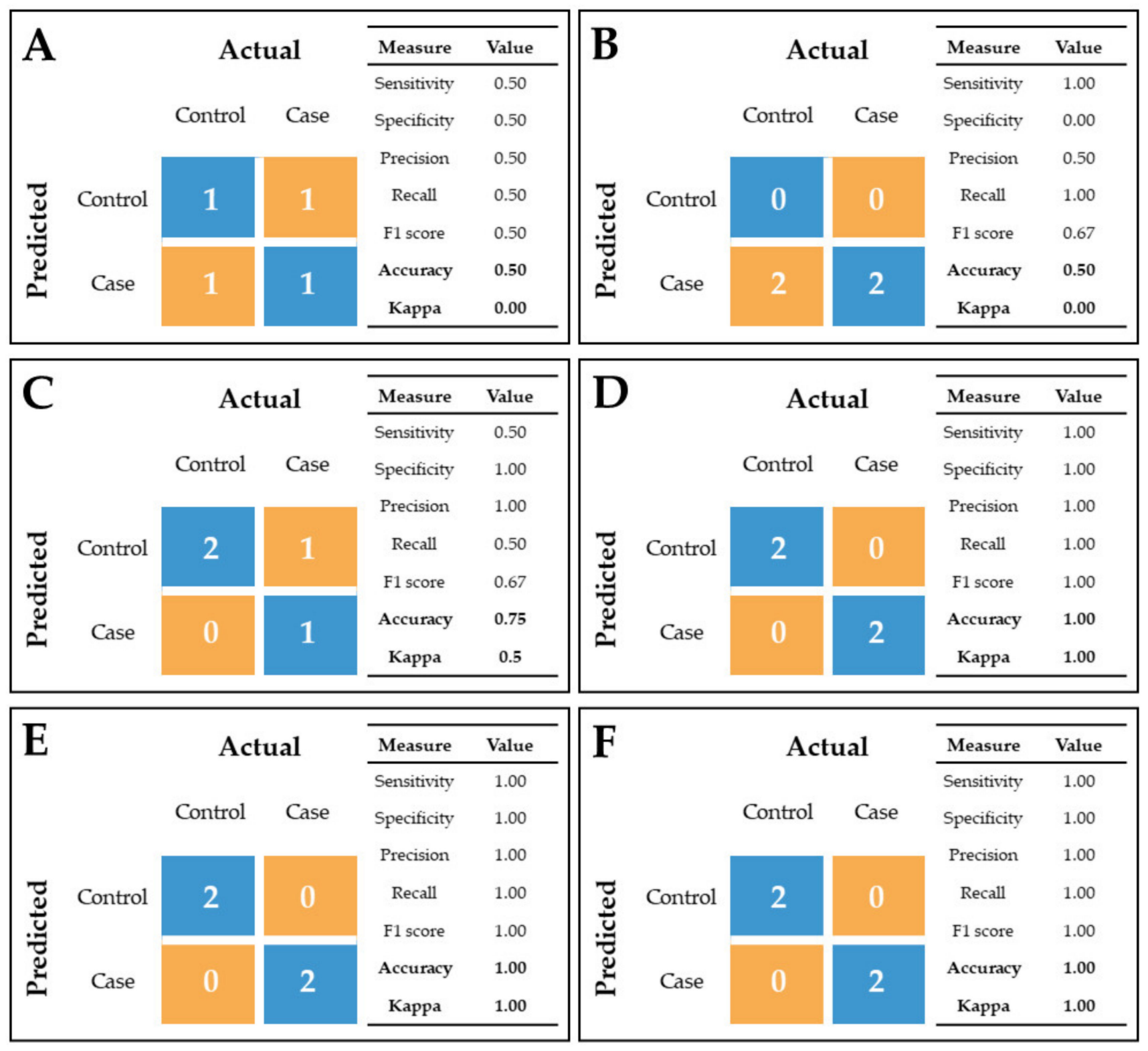

Figure 5. Performance of different classification models on test data using different algorithms and options for feature selection in the form of classification matrices: (A) minimal-redundancy-maximal-relevance (mRMR)-10 top-ranked features; (B) mRMR-20 top-ranked features; (C) mRMR-30 top-ranked features, (D) recursive feature elimination (RFE)-7 features selected; (E) RFE-9 features selected; (F) Combined mRMR (30 top-ranked features) and RFE (7 and 9 features selected)-only features in overlap in $2 / 3$ models were used (6 features).

Furthermore, to obtain the biological significance of our omics data, dual (metabolite and protein) pathway analysis was performed using all significant proteomic and metabolomic features. Both joint pathways that can be observed in the CSF due to IVDH are shown in Table 5, Figure 6a and Supplementary File S5. Furthermore, the pathwaybased network of significant features was also visualized by Metascape (Figure 6b). 
Table 5. KEGG pathways affected by intervertebral disc herniation in dogs observed in cerebrospinal fluid as obtained using MetaboAnalyst taking into consideration significantly deregulated proteins and metabolites.

\begin{tabular}{cccc}
\hline KEGG Pathway Name & $\begin{array}{c}\text { Total Features } \\
\text { in Pathway }\end{array}$ & $p /$ FDR * & Impact * \\
\hline Complement and coagulation cascades & 80 & $5.83 \times 10^{-2}$ & 0.3881 \\
Cholesterol metabolism & 60 & $1.99 \times 10^{-4}$ & 0.0583 \\
D-Glutamine and D-glutamate metabolism & 18 & $7.86 \times 10^{-3}$ & 0.3125 \\
Glycine, serine and threonine metabolism & 90 & $7.86 \times 10^{-3}$ & 0.1059 \\
Glyoxylate and dicarboxylate metabolism & 92 & $7.86 \times 10^{-3}$ & 0.1136 \\
Prion diseases & 38 & $4.54 \times 10^{-2}$ & 0.0313 \\
Mineral absorption & 87 & $4.87 \times 10^{-2}$ & 0.0118 \\
\hline
\end{tabular}

* The $p$-value after false discovery rate (FDR) correction was calculated during the pathway enrichment analysis in while the pathway impact value was calculated from the pathway topology analysis in MetaboAnalyst.
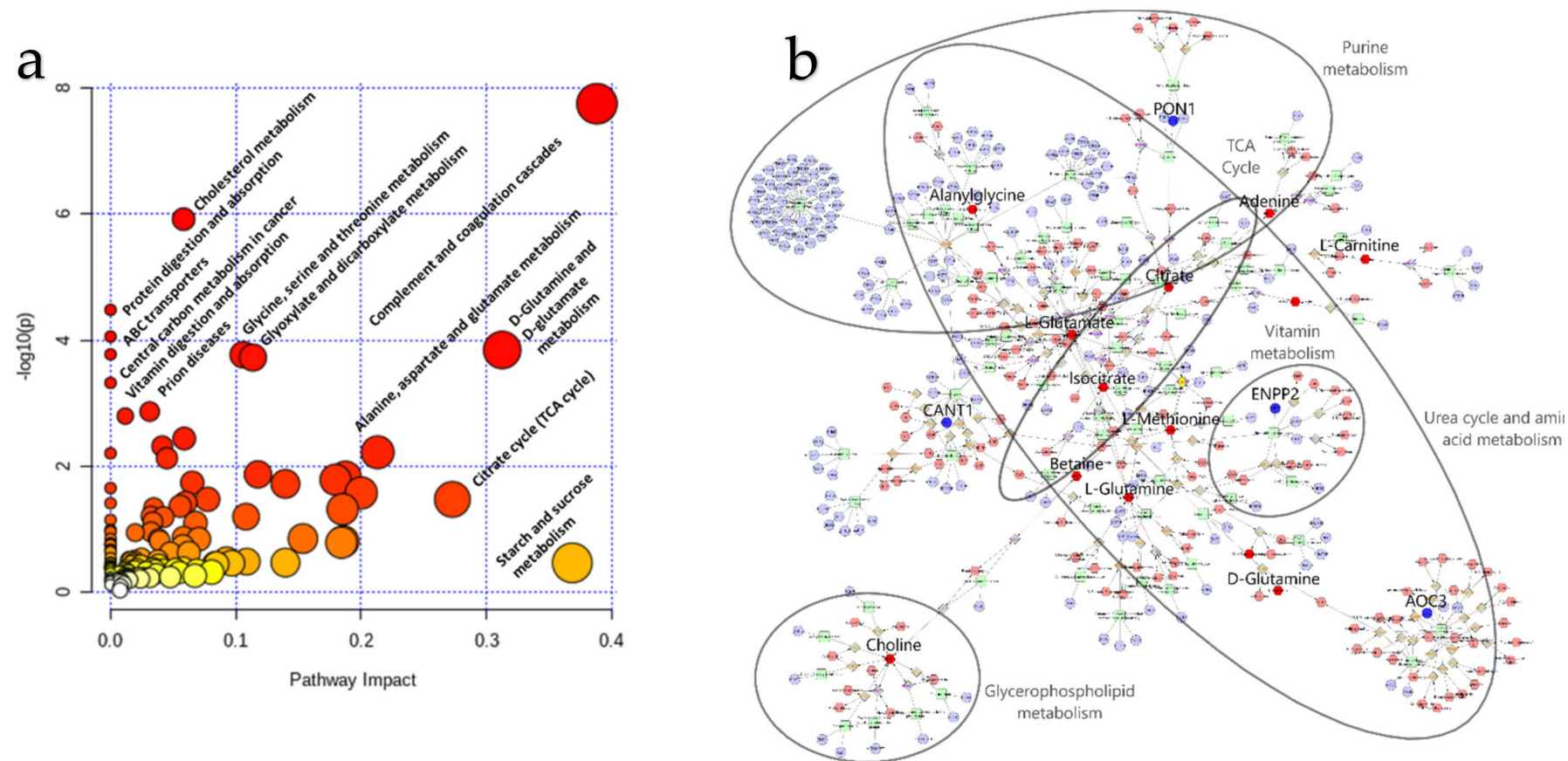

Figure 6. (a) Joint pathway enrichment analysis performed in MetaboAnalyst reveals the key pathways in cerebrospinal fluid affected by intervertebral disc herniation (IVDH) in dogs. (b) Pathway-based Compound-Reaction-Enzyme-Gene (C-R-E-G) network obtained using significantly deregulated genes and metabolites visualized in Cytoscape plugin Metscape (nods: blue-gene, red—compound/metabolite, green—enzyme). Highlighted blue (gene) and red (metabolite) nods represent input data. Enlarged feature names, together with the metabolic pathways, represent the features closely related to the IVDH. The C-R-E-G network is also available as a Supplementary File for better insight into various features (Supplementary Figure S9).

\section{Discussion}

Spine-related disorders represent significant health concerns for both humans and dogs, affecting the quality of life. Histologic, biomechanical, compositional and clinical similarities have enabled comparative studies between these species, making the canine IVDH model tremendously useful for both understanding the pathophysiology of disease and validating novel therapies prior to clinical trials [19]. The degenerative disc changes are commonly diagnosed by magnetic resonance imaging (MRI), X-ray or computed tomography (CT) scans [20]. Furthermore, total protein concentration elevation in CSF partially reflects the functional status of the nerve root (e.g., nerve root compression), leaking of plasma proteins through the blood-nerve root barrier into the CSF or can 
be indicative of a tumor [21,22]. However, the total protein concentration difference is mostly not significant in the CSF of IVDH dogs, in line with our previous research [22]. Finally, the pathogenesis involves multiple molecular events that cannot be identified by applying the above-mentioned methods and requires a more advanced systems approach. Understanding those molecular events may provide a better understanding of imbalances which could lead to more effective personalized treatments $[20,23]$. To obtain an insight into the complex biological processes in IVDH occurring at the molecular level, we used the novel integrative multi-omics approach, involving the integration of high-resolution mass spectrometry-based proteomics and global metabolome profiling data, which has not previously been applied in IVDH in human or veterinary medicine in IVDH. In relation to this current topic, and according to the best authors' knowledge, IVDH-related proteomic studies have been published to address quantitative analyses of IVD [24], secretome and proteoglycan in canine NP [25], as well as gel-based approaches in human CSF in IVDH [26]. Although there are recent papers reporting multi-omics (e.g., proteomics, metabolomics, transcriptomics and genomics) studies contributing to the identification of potential biomarkers in neurovascular diseases [27], the use of biostatistical omics data integration is still at an early stage [28].

In our study, the LC-MS/MS analysis of TMT-labelled CSF tryptic peptides enabled the identification of 73 master proteins involved in calcium binding, lipid, vitamin and amino acid metabolisms as well as immune response, presenting the potential protein biomarker candidates of IVDH. This information, although valuable for further potential clinical diagnostic application, requires further studies and clinical validation. Accordingly, it can be expected that some proteins, with regards to their abundance in the CSF, are showing limited biological intra-individual variations in the CSF due to age and gender (proteins that originate from blood, serotransferrin, vitamin D-binding protein, ganglioside GM2 activator, beta-2-glycoprotein) unlike inflammatory proteins and proteins specific to CNS in humans [29]. Based on the PCA plot (Supplementary Figure S1), it can be observed that global proteomic profiles of selected individual animals within groups are not so consistent; however, they are clearly separated from the control group. It can be concluded accordingly that the changes in abundance are at their highest extent caused by the disease.

MS results presented herein were analytically validated by quantitative measurements of albumin concentration and enzyme activity of PON1 in the CSF of IVDH dogs compared to the control group, where the CSF of five dogs with IVDH (not previously used for omics analyses) and the CSF of six healthy dogs were used. The proteins used for validation were selected based on both biological importance and test availability. Albumin is the major protein in CSF, exclusively produced by the liver; thus, its concentration in CSF depends on the blood-brain barrier (BBB). If total protein concentration in the CSF increases, albumin concentration increases disproportionally due to molecular size and blood-brain barrier (BBB) disruption. Since albumin was found to be abundant in the CSF of human patients following incomplete spinal cord injury at 1-8 days post-injury, being a potential biomarker for spinal cord injury progression and recovery [30], it was selected for analytical validation. In our study, elevated concentration of albumin in the CSF of IVDH group revealed by TMT label-based proteomics was also confirmed by using immunoturbidimetric assay (Figure 3). The antioxidant PON1 has an anti-inflammatory role and was documented in the serum, CSF, and brain, suggesting its activity in neurodegenerative disease. Furthermore, PON1 plays an important role in keeping the homeostatic balance of intervertebral disc and for this reason, it was selected for analytical validation. Unlike in our results, CSF PON1 activity was reported to decrease with the progression of neurodegenerative diseases and Alzheimer's disease, as well as dementia [18]. However, plasma PON1 activity in animal models of spinal cord injury increases [31] and the cases studied in this investigation were more related to this type of injury rather than degeneration and lead to an increase in PON1. Increased levels of PON1 and its activity can be explained by PON1 having a protective role in the early phase of oxidative stress because of antioxidant enzyme consumption [31]. 
The increase in CSF PON1 activity was also obtained by assay, using p-nitrophenyl acetate as a substrate herein, confirming the proteomic findings.

The CSF metabolomics profile mainly reflects brain metabolic processes but also consists of intermediate and end products of neurotransmission and energy metabolism. Among others, metabolic changes in CSF can be caused by various pathophysiological processes, such as inflammation or response to oxidative stress responses [32], which was also the case in IVDH dogs in our study. To fill the existing gaps in IVDH-related knowledge, it is necessary to obtain detailed insight into the metabolomic profile. We are aware that due to metabolite diversity, it is impossible to cover all metabolites by a single method and/or using only one analytical platform, and this is probably the reason why some, for example, lipid metabolites related to lipid peroxidation, neuroinflammation and changes in antioxidant compounds like glutathione [32,33] were not identified/detected as changed in the CSF herein. However, although known IVDH-related important metabolites might be absent, pathway analysis revealed that C5-Branched dibasic acid metabolism, D-Glutamine and D-glutamate metabolism, Arginine and proline metabolism, Glutathione metabolism and Nitrogen metabolism are significantly affected by IVDH. All above-mentioned pathways were reported significantly affected in spinal cord damage, including demyelination in multiple sclerosis [33]. Branched-chain amino acids are crucial for brain metabolism and function as they are an important source of pyruvate in energy metabolism and serve as nitrogen donors in protein synthesis [34]. Arginine metabolism, nitrogen metabolism and glutathione metabolism are linked to oxidative stress. Arginine serves as a precursor to nitric oxide and changes within arginine metabolism might result in the synthesis of nitric oxide. The formation of reactive nitrogen species is cytotoxic for nerves, promoting myelin destruction [34]. Although we did not find the glutathione itself to be aberrant, as a protective compound against oxidative stress by oxidizing to glutathione disulfide (GSSG) with a simultaneous reduction of $\mathrm{H} 2 \mathrm{O} 2$, the concentration alliteration of metabolites such as pyroglutamic acid [35] revealed the deregulation of glutathione metabolism in IVDH. The elevation of pyroglutamic acid has not yet been related to IVDH, unlike anion-gap metabolic acidosis [36] or cancer [37], according to the authors' knowledge. Affected metabolic pathways and the alliteration of antioxidant defense molecules such as an increase in superoxide dismutase type 1 (SOD1) imply that oxidative stress may also play an important role in IVDH. IVDH-affected pathways in the CSF also included D-glutamine and D-glutamate metabolism, and glutamate was also found to be significantly elevated in IVDH dogs. Glutamate is the major excitatory neurotransmitter in the CNS and its extracellular accumulation points out the abnormalities in the glutamatergic neurotransmission [38]. Elevated concentrations of glutamate have catastrophic impacts on nervous tissue due to the overstimulation of glutamate receptors, which can lead to subsequent excitotoxic injury of glial cells and neurons [39]. The increase of glutamate in the CSF has been related to several neurological and brain pathologies such as multiple sclerosis, Alzheimer's and Parkinson's disease or during ischemia [39].

\subsection{Integromics Model Reveals Key Molecules for IVDH}

The main task of omics data integration, besides obtaining knowledge about disease using various omics layers, is to be able to straightforwardly classify patients with a high specificity based on the best omics feature selection. For our pilot study, the challenge was to use a well-defined (as can be seen from the omics PCA plots, Figures 1 and 3) but small population size with multiple proteomic and metabolomic features for the selection of the most suitable data-driven and data-mining tools in order to define the pathways and the CSF features best representing the IVDH in dogs that can be used for disease diagnosis, monitoring and/or progression. Data-driven statistical methods integrate multi-omics data sets performing sample clustering or classification, equalizing the contribution of different omics data which could ignore biological relationships between different types of molecules [17]. Since better performance can be achieved by combining several selection algorithms [40], we used RFE and mRMR feature selection algorithms to integrate the 
analysis of multi-omics data addressing three major challenges [41], specifically the effect of dimensionality, the differences in scales, and extracting the most relevant features across different multi-omics results. mRMR and RFE algorithms have been applied widely in omics analyses of gene expression data for glioblastoma prognosis [42] and cancer classification [43].

In our study, the six most significant omics features selected by the optimal classification model were FSTL1, SCG5, NUCB1, CRSP2, N-acetyl-D-glucosamine and adenine, respectively. However, their biological role should be determined by data mining. Data mining computational tools derive pathways and molecular interactions from the correlation structures, which provides the results with the biological significance [17]. However, biological knowledge (molecular interactions and pathway data) is under permanent change by data curators due to new data generation and is thus biased, with the density of connections in certain regions of the network affected by (in)completeness of experimentally proven molecular interactions and their availability within databases [42]. To determine the effectiveness of our classification model, data mining of biological significance and the feature's potential relation to IVDH revealed FSTL1, SCG and CRSP2 as key molecules in studies addressing neuropathic pain [44]. Furthermore, NUCB1 was reported as a novel pan-neuronal calcium handling marker, correlated to Alzheimer's disease [45]. Unlike those, $\mathrm{N}$-acetyl-D-glucosamine has a neuroprotective role as a regulator of primary myelination and myelin repair and is of importance in demyelinating diseases like multiple sclerosis [46]. Finally, adenine is, as one of the purines, involved in neuromodulation, neurotransmission, activation of differentiation and neuritogenesis of precursor cells and neurons. Additionally, purines also contribute to immunological response due to astrocytes and microglia activation, initiating the inflammatory reactions [46]. The diagnostic significance and potential are yet to be examined by further studies.

\subsection{Integrative Omics Analysis Reveals Key Processes Referring to IVDH}

Although not easily accessible as serum or saliva due to technical and ethical reasons (especially in the case of healthy individuals), CSF was proven to be a very informative biofluid for omics analyses. Herein, by using an integromics approach of proteomic and metabolomic data, we demonstrated that a plethora of events occurs in IVDH in dogs, that can be observed by CSF analyses at the molecular level (Figure 7). Multiple features were found, revealing the most common anomaly - a manifestation of degenerative changes in terms of collagen (COL1) degradation, causing IVDH, which may result in nerve damage, spinal cord injury (such as deficiency of apolipoprotein E (APO E)) [47] and neuropathic pain (such as a higher representation of mimecan and/or apolipoprotein C1) [48].

Annulus fibrosus, the outer layer of intervertebral disc, is, together with elastin, fibroblast-like cells and proteoglycans composed of collagen type I [3]. Collagen is a biological polymer continually synthesized and degraded in the extracellular space. However, among dogs with IVDH, excessive collagenolysis has been demonstrated in diseases such as atherosclerosis, arthritis and even cancer. Collagen is composed of tropocollagen, containing repeating triplets of amino acids $X 1, X 2$ and glycine $(X 1-X 2-G)$, with many $X 1 / X 2$ occupied by either proline or hydroxyproline [49]. Integrated omics and metabolomic analyses emphasized the glycine, serine and threonine metabolism pathways as significantly affected by IVDH, with 17 annotated and 7 identified metabolites ( $45 \%$ coverage, as shown in Table 3), as well as the arginine and proline metabolism pathways having 31 annotated and 7 identified metabolites ( $42 \%$ coverage, as shown in Table 3$)$. In our study, 4-hydroxyproline was found to be elevated in the CSF of the IVDH group, showing collagen degradation [50]. Following annotation based on molecular ion $\mathrm{m} / \mathrm{z}$ and fragment ions (Supplementary File S4), other collagen fragments presented as series metabolites isoleucyl-proline, glycyl-hydroxyproline and valyl-glycine, and were also found to be different in concentration in the IVDH group (however, not significantly according to our results). Unlike previous metabolomic studies of collagen degradation-related diseases in nerve, serum and/or urine, other collagen degradation products such as amino acids 
(glycine, proline, alanine, leucine and valine) were not found to be significantly changed in this study, which might be related to the analytical method/platform selectivity or degree of disc degradation [50-52]. Interestingly, the statistically lower abundance of collagen (COL1) was also found in the CSF of dogs in the IVDH group, which might be explained by collagen deposition during wound healing [53].

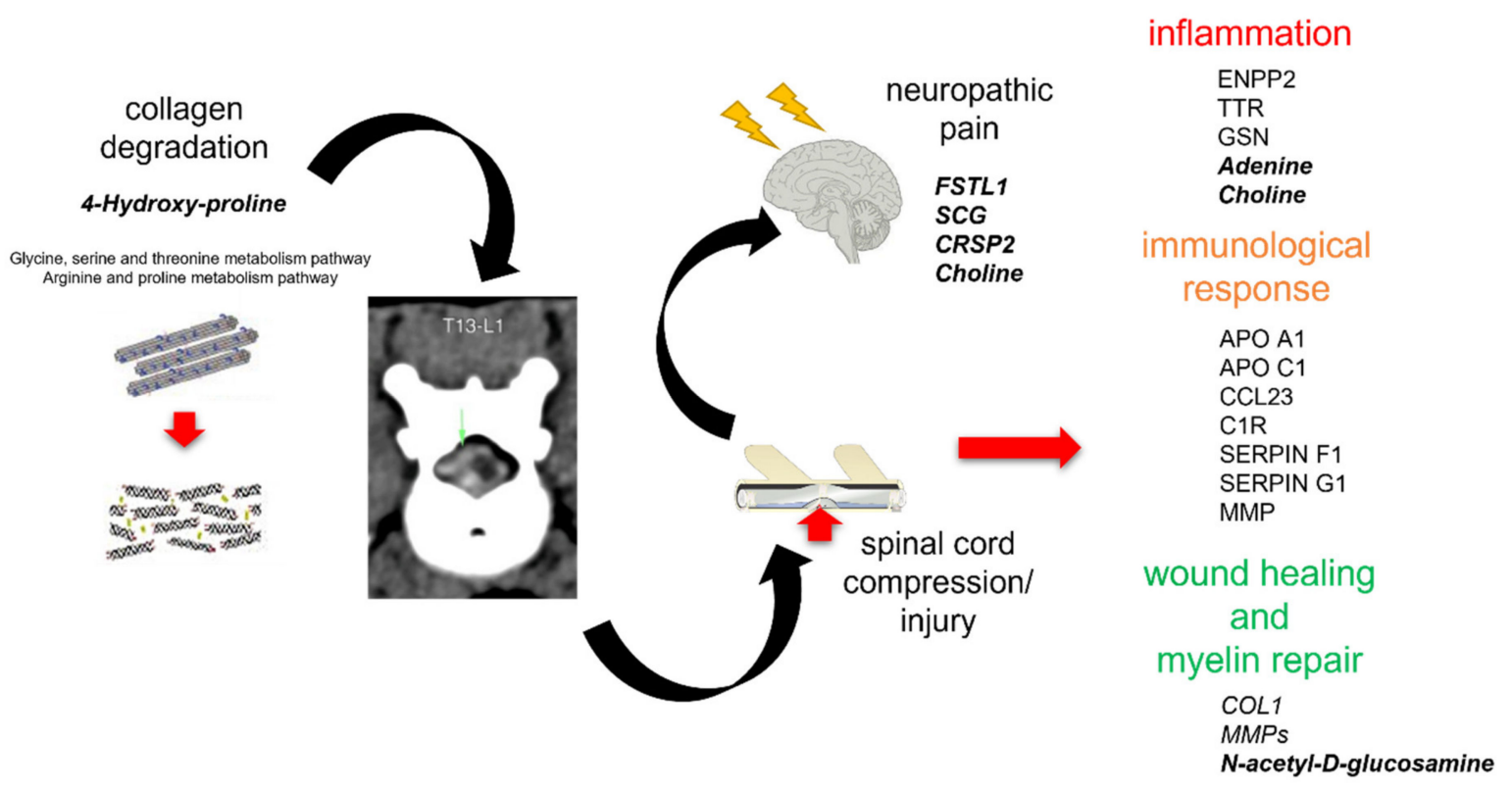

Figure 7. Complex processes occurring in cerebrospinal fluid of dogs with intervertebral disc herniation (IVDH) revealed by integrative omics approach pointing out the key molecules (in bold) proposed by our classification data-driven model. Related proteins: follistatin Like 1 (FSTL1), calcitonin receptor-stimulating peptide 2 precursor (CRSP2), secretogranin (SCG), nucleobindin 1 (NUCB1), Ectonucleotide Pyrophosphatase/Phosphodiesterase 2 (ENPP2), transthyretin (TTR), gelsolin (GSN), apolipoproteins (APO A1 and APO C1), C-C Motif Chemokine Ligand 23 (CCL23), Complement C1r (C1R), Serpins (SERPIN F1 and G1), matrix metalloproteinases (MMP) and collagen 1 (COL1).

When collagen degrades, the fibrous ring ruptures and the protrusion of the nucleus pulposus can press on a nerve root and/or the spinal cord, bursting the nucleus of the disc and damaging the nerve and compressing the spinal cord. Interestingly, unlike altered proteins related to spinal cord injury (transferrin-TF, matrix metalloproteinases-MMPs, insulin-like growth factor 1-IGF-1 and apolipoprotein E-APO E) [54,55], no metabolites reported in both untargeted and targeted metabolomics SCI-related studies [56] were found to be altered. This might be explained by the severity of SCI itself in the IVDH group, which was mild. However, we found evidence of wound-healing events such as myelination and myelin repair (N-acetyl-D-glucosamine, MMPs), angiogenesis and neurogenesis (MMPs). Due to spinal cord compression and nerve injury, inflammation and/or ischemia occur, which are important processes of disc herniation. Changes in inflammation markers were associated with proteins such as APO A1, C-C Motif Chemokine Ligand 23 (CCL23) and MMP2. APO A1 is the principal protein fraction of high-density lipoproteins (HDL), involved in reverse cholesterol traffic, as well as inflammatory and immune response. It has a protective role against cardiovascular diseases and neuroinflammation in neurodegenerative diseases [57]. Furthermore, CCL23 was found to be deranged in the CSF in our study. Unlike some other studies addressing neuroinflammation where it was increased, albeit not significantly [58], neuroinflammation was in contrast found to be decreased in our study. CCL23 is a chemokine- a circulating and tissue-inflammatory molecule, being a chemotactic factor for monocytes/macrophages, dendritic cells and lymphocytes, up-regulating proinflammatory cytokine release. CCL23 was proposed to be a blood biomarker for the early diagnosis of cerebral damage [59]. CCL23 is expressed in response to IL-4 and LPS, TNF $\alpha$ and IFN- $\gamma$ and signals through CCR1 [60]. In our case, the patients had acute IVDH, 
and the enzymatic cascade enabling the release of CCL23 was probably not yet activated. Matrix metalloproteinases (MMPs) are involved in the pathogenesis of neuroinflammatory diseases [61]. MMP2 (72-kDa gelatinase A) can be found in a healthy brain, in neurons from the cortex, cerebellum and CSF. It was found to be decreased in the CSF of the IVDH group, as reported in neuroinflammation [62]. Additionally, MMP2 specifically cleaves type IV collagen, the major structural component of basement membranes, increasing the permeability of BBB. MMP-2 is also important in remyelination, angiogenesis and neurogenesis [62]. Inflammation processes caused by cell injury/tissue damage initiate both innate and adaptive immune responses, which was also determined in our study [63]. Multiple proteins already documented as being involved in the maintenance of CNS and immune surveillance against injured cells have been altered in IVDH dogs; for instance, complement C1R, whose activity is tightly regulated to protect host cells, and its regulator SERPIN G1 (which binds to and inactivates $\mathrm{C} 1 \mathrm{r}, \mathrm{C} 1 \mathrm{~s}$ and MASP-1/2 proteases) [64]. Lower levels of serpin protein $\mathrm{C} 1$ inhibitor activate kinin pathways and increase levels of bradykinin in the blood, which is responsible for capillary leakage and angioedema [65]. Cell injury (tissue damage), nerve root compression and swelling, as well as disc inflammation, may have an important role in pain generation. The bioinformatic analysis of CSF proteins related to neuropathic pain gathered from multiple studies [66] identified three groups of proteins involved in inflammatory responses (APO A1, APO C1 [49], SERPIN F1), immune responses (ectonucleotide pyrophosphatase/phosphodiesterase 2 (ENPP2), transthyretin (TTR), gelsolin (GSN)) and metabolic processes (albumin, prothrombin (F2)), and these were all found changed in our study. The application of metabolomics in pain research is still at an early stage [67]. Notwithstanding this issue, the metabolomics of the CSF in pain patients revealed that neuropathic pain alters sphingomyelin-ceramide metabolism, nervous tissue damage (alanine, taurine) and inflammatory processes (choline) [67-69]. Choline, which was found to be elevated in IVDH dogs in our study, is a metabolite related to glial activity, and its elevation was found to be linked with worse pain interference [69].

Our pilot study using canine clinical samples was constrained by sample size restrictions (especially in the control group due to animal ethical guidelines), as well as CSF sample volume availability, testing research hypotheses in a small number of subjects. Furthermore, ideal matching based on canine age and the weight of the control group compared to the study group was not possible for operational reasons (especially related to owners' consent for CSF collection in healthy dogs and ethical guidelines). However, our findings are considered to be a valid indication of the change in CSF due to intervertebral disc herniation.

\section{Materials and Methods}

\subsection{Experimental Design}

A total of 14 client-owned dogs presented to the veterinary clinics of the Faculty of Veterinary Medicine, University of Zagreb, Croatia, as well as 6 shelter dogs were involved in this pilot study. The sample size was determined based on recommendations for statistical consideration of optimal clinical study design in proteomics in preliminary studies for biomarker discovery [70,71], taking into consideration gender, age, sample type, quantification method (isobaric labeling) and availability of samples due to ethical restrictions (it is ethically not acceptable to collect CSF in healthy dogs for the purpose of scientific research, only because the procedure of sampling requires general anesthesia and cerebellomedullary puncture, which is an invasive method). The current pilot study was provided to patients who were treated at the Veterinary Faculty, University of Zagreb. The owners signed a written consent for participation in the study on the acceptance of the risk of anesthesia and surgical procedure. All dogs were submitted for a general physical examination, and general hematology was performed. Prior to general anesthesia, pre-anesthetic assessment of patients was performed.

The omics studies involved canine patients classified into two groups. Healthy dogs (control group) $(\mathrm{N}=6)$ were mixed breeds (20 kg average body weight), age 2-6 years, 
while the intervertebral disc herniation group $(\mathrm{N}=9)$ were mixed breeds (body weight 6-15 kg), age 6-12 years. In IVDH dogs, disc herniation was detected in T13/L1/L2 discs by CT. The dogs in IVDH group were paraplegic with preserved deep pain reflex and underwent a surgical procedure for decompression of the spinal cord. Additionally, 5 samples from IVDH group not used for omics studies were randomly selected for the analytical validation (age 6-12 years). The Ethical Committee at the Faculty of Veterinary Medicine, University of Zagreb, Croatia approved the research (No. 640-01/18-17/67; Record No. 251-61-21/333-18-01).

\subsection{Sample Collection}

Cerebrospinal fluid was sampled under general anesthesia from cisterna magna prior to operation. Induction to general anesthesia was obtained with propofol (Propofol®, Abbott, Maidenhead, UK), administered intravenously and maintained with sevoflurane (Sevorane ${ }^{\circledR}$, Abbott, Maidenhead, UK) applied through an endotracheal tube. Anesthesia was adjusted to minimally interfere with cerebral blood flow and intracranial pressure [72]. The CSF was collected into sterile Eppendorf tubes (size $1.5 \mathrm{~mL}$ ), immediately centrifuged at $3000 \times g$ at $4{ }^{\circ} \mathrm{C}$ for $5 \mathrm{~min}$, aliquoted into Eppendorf tubes (size $1.5 \mathrm{~mL}$ ) to avoid multiple freeze-thaw cycles and stored at $-80^{\circ} \mathrm{C}$ till analyzed. All samples were collected within 6 months.

\subsection{Proteomic Analysis}

Proteomic analysis of CSF samples (IVDH N $=9$ and control $\mathrm{N}=6$ ) was performed by using a gel-free TMT-based quantitative approach, as described previously [18]. In short, total protein concentration was determined using a bicinchoninic acid assay (BCA assay) (Thermo Scientific, Rockford, IL, USA). An internal standard was prepared by mixing equal protein amounts of each CSF sample. An amount of $35 \mu \mathrm{g}$ CSF proteins was diluted to a volume of $100 \mu \mathrm{L}$ using $0.1 \mathrm{M}$ triethylammonium bicarbonate (TEAB, Thermo Scientific, Rockford, IL, USA), reduced ( $5 \mu \mathrm{L}$ of $200 \mathrm{mM}$ dithiothreitol for $60 \mathrm{~min}, 55^{\circ} \mathrm{C}$ ) (Sigma Aldrich, St. Louis, MO, USA), alkylated ( $5 \mu \mathrm{L}$ of $375 \mathrm{mM}$ IAA at $30 \mathrm{~min}$, room temperature in the dark) (Sigma Aldrich, St. Lois, MO, USA) and acetone-precipitated $(600 \mu \mathrm{L}$, overnight, $-20^{\circ} \mathrm{C}$ ) (Merck, Darmstadt, Germany). The obtained protein pellets were dissolved in $50 \mu \mathrm{L}$ of $0.1 \mathrm{M} \mathrm{TEAB}$ and digested using trypsin gold $(1 \mathrm{mg} / \mathrm{mL}$, Promega; trypsinto-protein ratio 1:35, at $37^{\circ} \mathrm{C}$ overnight). For labeling, TMT tenplex reagents (Thermo Scientific, Rockford, IL, USA) were prepared as recommended by manufacturers and $19 \mu \mathrm{L}$ of each reagent were used for labeling. The internal standard was labeled with TMT $m / z$ 131, while other samples were randomized within two TMT experiments. Labeling reaction was quenched by $5 \%$ hydroxylamine (Sigma-Aldrich, St. Louis, MO, USA). Per experiment, nine TMT-labelled peptide samples were combined with the internal standard, aliquoted, dried and stored at $-80^{\circ} \mathrm{C}$ for subsequent LC-MS/MS analysis.

High-resolution LC-MS/MS analysis was performed using an Ultimate 3000 RSLCnano system (Dionex, Germering, Germany) coupled with a Q Exactive Plus mass spectrometer (Thermo Fisher Scientific, Bremen, Germany) as reported elsewhere [18]. Differentially TMT-labelled peptides were desalted on the trap column and separated on the analytical column (PepMap ${ }^{\mathrm{TM}}$ RSLC C18, $50 \mathrm{~cm} \times 75 \mu \mathrm{m}$, Thermo Scientific, Rockford, IL, USA) using linear-gradient $5-45 \%$ mobile phase B $(0.1 \%$ formic acid in $80 \% \mathrm{ACN})$ over $120 \mathrm{~min}$, at the flow rate of $300 \mathrm{~nL} / \mathrm{min}$. Mobile phase A consisted of $0.1 \%$ formic acid in LC-MS-grade water. Ionization was achieved using a nanospray Flex ion source (Thermo Fisher Scientific, Bremen, Germany) equipped with a $10 \mu \mathrm{m}$-inner diameter SilicaTip emitter (New Objective, Littleton, MA, USA). The MS operated in positive ion mode using DDA Top8 method. Full scan MS spectra were acquired in range from $m / z 350.0$ to $m / z 1800.0$ with a resolution of 70,000, $120 \mathrm{~ms}$ injection time, AGC target 1E6, a \pm 2.0 Da isolation window and the dynamic exclusion $30 \mathrm{~s}$. For HCD fragmentation, step collision energy $(29 \%$ and $35 \%$ NCE) with a resolution of 17,500 and AGC target of 2E5 were used. Precursor ions with 
charge states of +1 and more than +7 , as well as unassigned charge states, were excluded from fragmentation.

For protein identification and reporter ion-based relative quantification, the SEQUEST algorithm implemented into Proteome Discoverer (version 2.3., Thermo Fisher Scientific, Bremen, Germany) was applied. Database search against Canis lupus familiaris FASTA files (downloaded from NCBI database 04/04/2019, 172,083 entries) was performed using parameters as follows: two trypsin missed-cleavage sites, precursor and fragment mass tolerances of $10 \mathrm{ppm}$ and $0.02 \mathrm{Da}$, respectively; carbamidomethyl (C) fixed peptide modification, oxidation $(\mathrm{M})$ and TMT sixplex (K, peptide $\mathrm{N}$-terminus) dynamic modifications. The false discovery rate (FDR) for peptide identification was calculated automatically based on decoy database by the Percolator algorithm within the Proteome Discoverer workflow and was set at $1 \%$. Proteins containing at least two unique peptides and 5\% FDR were reported as identified. The mass spectrometry proteomics data were deposited to the Consortium via the PRIDE partner [73] repository with the dataset identifier PXD024921.

\subsection{Analytical Validation of Proteomic Results}

Frozen aliquots of CSF (IVDH N $=5$ and control $\mathrm{N}=6$ ) were thawed to determine paraoxonase 1 (PON1) activity, as well as albumin (ALB) concentration using automated biochemistry analyzer Abbott Architect c4000 (Abbott, Chicago, IL, USA). PON1 activity was assayed using the method of Tvarijonaviciute $e t$ al. with p-nitrophenyl acetate (SigmaAldrich, Saint Louis, MO, USA) as substrate [74]. Albumin concentration in CSF samples was evaluated with human immunoturbidimetric assay (Microalbumin OSR6167 Beckman Coulter, Brea, CA, USA) which was previously validated for use in canine CSF samples [75].

\subsection{Untargeted Metabolomic Analysis}

Metabolomics analysis of CSF samples (IVDH N = 7 and control $\mathrm{N}=6$ ) was performed using an untargeted approach, as described [76]. In short, pooled sample was prepared by mixing $10 \mu \mathrm{L}$ of each sample (control and IVDH). Matrix blank sample contained extraction solvent. For metabolite extraction, a volume of $25 \mu \mathrm{L}$ of each sample was subjected to chloroform/methanol/water (1:3:1, $v / v / v)$ extraction on a vortex mixer for two hours at $4{ }^{\circ} \mathrm{C}$. All samples (CSF samples, pooled sample, matrix blank) were subsequently centrifuged $\left(13,000 \times g\right.$ for 5 min at $\left.4{ }^{\circ} \mathrm{C}\right)$. The supernatant $(200 \mu \mathrm{L})$ was stored at $-80{ }^{\circ} \mathrm{C}$ until LC-MS/MS analysis.

Metabolite extracts were separated on a $4.6 \mathrm{~mm} \times 150 \mathrm{~mm}$ zwitterionic-polymeric hydrophilic interaction chromatography (ZIC-pHILIC) column (Merck SeQuant, Darmstad, Germany) using Dionex UltiMate 3000 RSLC system (Dionex, Germering, Germany) coupled to a Thermo Orbitrap Q Exactive Plus (Thermo Fisher Scientific, Hemel Hempstead, UK) using a gradient of $80 \%$ to $95 \%$ of mobile phase A for $15 \mathrm{~min}$ at $25^{\circ} \mathrm{C}$ and a flow rate of $0.3 \mathrm{~mL} / \mathrm{min}$, as reported [76]. Mobile phase A contained $20 \mathrm{mM}$ ammonium carbonate in water and B contained $100 \%$ acetonitrile. The injection volume was $10 \mu \mathrm{L}$. Samples were maintained in the autosampler at $5{ }^{\circ} \mathrm{C}$ prior to injection. The mass spectrometer was operated by altering positive and negative modes with electrospray ionization at a resolution of 70,000 and the full scan $m / z$ range of 70-1050. The MS settings for positive electrospray ionization were used as follows: source voltage of $3.8 \mathrm{kV}$, sheath gas 40 (arbitrary units), auxiliary gas of 5 (arbitrary units) and capillary temperature of $320^{\circ} \mathrm{C}$. A standard mix containing a mix of 148 authentic compounds-metabolites-was used for metabolite identification. A standard mix (containing reference compounds for metabolite identification) and quality control samples used in metabolomics analysis herein were kindly donated by Dr. Richard Burchmore, Glasgow Polyomics, College of Medical, Veterinary and Life Sciences, University of Glasgow, UK. Metabolomics data were deposited to the EMBL-EBI MetaboLights [77] database (DOI: 10.1093/nar/gkz1019, PMID:31691833) with the identifier MTBLS2689.

Metabolomics data were analyzed using the Polyomics integrated Metabolomics Pipeline (PiMP), available at http:/ / polyomics.mvls.gla.ac.uk (accessed on 14 January 
2021), using standard workflow and default parameters [78]. In short, raw data was converted from the 'RAW' file format (Thermo Scientific) to an open-source ' $m z X M L$ ' file format, centroided and split into positive and negative polarities using MSConvert tool [79]. Metabolite identification was performed in PiMP, following Metabolite Standards Initiative guidelines by matching retention times and accurate masses of detected peaks with either the authentic standards or were annotated (assigned putatively based on accurate masses) using metabolite libraries search (e.g., The Human Metabolome Database, HMDB, and/or Kyoto Encyclopedia of Genes and Genomes, KEGG) integrated within PiMP standard workflow. The metabolic maps provided within PiMP software were derived from the KEGG database.

\subsection{Statistical and Bioinformatic Analyses \\ 4.6.1. Proteomics}

Proteins that had more than $50 \%$ missing values were excluded from the subsequent statistical analysis. Statistics were performed using R v3.2.2 [80]. Sample outliers were detected per each group for each protein using Dixon's test from $R$ package outliers v0.14 [81]. Significant outliers $(p<0.05)$ were removed from further analysis. To test the difference in protein abundance between groups, Mann-Whitney U test was performed. Fold change (FC) between two groups was calculated as mean(IVDH)/mean(control) and expressed on log2 scale. PCA and volcano plots were designed using R package ggplot2 v3.1.1 [82].

For bioinformatics, proteins GI accession numbers were converted into official gene symbol by DAVID conversion tool (https:/ / david.ncifcrf.gov/conversion.jsp, accessed on 19 March 2021) and UniProtKB ID mapping (https:/ / www.uniprot.org/uploadlists/, accessed on 19 March 2021). Protein-protein interactions and REACTOME pathway analysis was performed using STRINGdb v11.0 (https:/ / string-db.org/, accessed on 26 March 2021) [83], with the selection of Canis lupus familiaris default settings with the exception of no more than 5 interactors to show in the 1st shell. Networks of relationship between REACTOME pathway and proteins with significantly differential abundances between groups were designed using Cytoscape v.3.7.1. (https:// cytoscape.org/, accessed on 10 June 2021) [84]. Annotation terms (GO Biological process, GO Molecular function, GO Cellular component) were generated using Database for Annotation, Visualization and Integrated Discovery (DAVID) v6.7. (https:/ / david.ncifcrf.gov/, accessed on 10 June 2021) [85].

\subsubsection{Proteomic Data Validation}

The significance of PON1 activity within CSF samples of healthy and IVDH dogs, as well as albumin concentrations in CSF, was determined by applying the Mann-Whitney U test within GraphPad Prism software (v.8.4.2.).

\subsubsection{Metabolomics}

Metabolomics raw data were submitted to the PiMP for metabolite annotation/ identification, statistical analysis and metabolic pathway analysis using a standard workflow [78]. One group-wise comparison was undertaken to identify differences between control and IVDH group (fold changes expressed as log2FC). Peaks with an (BenjaminiHochberg) adjusted $p$-value less than 0.05 were considered significant. Further analyses and visualization of PiMP-exported data (peak intensities, $\log 2 \mathrm{FC}$ and FDR values of identified/annotated metabolites) were performed using R v.3.2.2. (volcano plot, PCA plot and heatmap) [80].

\subsubsection{Statistical Omics Data Integration}

Significantly altered and identified metabolites, as well as significantly altered proteins related to unique genes were merged to build a classification model where only the most associated proteomic and metabolic features would be able to discriminate between healthy 
dogs and dogs diagnosed with disc herniation. First, samples that have both proteomic and metabolomic data (6 controls and 7 IVDH samples) were randomly divided into training and test set in a 70:30 ratio, leading to 9 samples in the training set and 4 samples in the test set. Metabolic data were first log-transformed for further use. The training set was then scaled, centered and the possible missing values were imputed using K-nearest neighbor imputation. We performed elastic net logistic regression with 5-repeated 10-fold crossvalidation to fit the training set. Features were then ranked according to their importance and the model was applied on the test set whose performance was evaluated with a confusion matrix. Due to the multicollinearity issue when dealing with small sample size and large number of integrated proteomic and metabolic features, we applied two feature selection algorithms to reduce the number of features to fit the model. One was recursive feature elimination (RFE) algorithm with 5-repeated 10-fold cross-validation and different numbers of features to retain the most important ones. The second one was minimalredundancy-maximal-relevance (mRMR) algorithm, which ranks features based on their correlation with the output but removes redundant features. For this, we considered the first 10, 20 and 30 ranked features. R packages caret [86] version 6.0-86, glmnet [87] version 4.0-2 and mRMRe [88] version 2.1.0 were used to build classification models.

\subsubsection{Bioinformatic Omics Data Integration}

Joint-pathway analysis of metabolites (compound name with matching fold changes used as input) and proteins (official gene symbol with matching fold changes used as input) that were significantly changed due to IVDH was conducted in MetaboAnalyst v.4.0. by applying Homo sapiens (human) as model organism. Further visualization was performed in Cytoscape plugin Metscape [89] v3.1.1. to identify enriched metabolic pathways. For the analysis, the lists of significantly changed genes and metabolites were imported into the Metscape, and human was set as a model organism, to obtain pathway-based CompoundReaction-Enzyme-Gene (C-R-E-G) network using standard workflow.

\section{Conclusions}

Complex conditions, such as IVDH, require a systems biology-based approach that can reveal not only progressive structural and functional changes of patients but also various pathophysiological processes that could be linked to the disease severity and patient's outcome. For that reason, we applied proteomic and metabolomic analyses combined with the state-of-the-art bioinformatics tools to obtain insight into the various molecules affected by IVDH. We set up a prediction model to examine the most important CSF features involved in IVDH with diagnostic potential and identified the key molecules, namely proteins FSTL1, SCG5, NUCB1 and CRSP2 and the metabolites N-acetyl-D-glucosamine and adenine, involved in neuropathic pain, myelination, neurotransmission and inflammatory response, respectively. Their clinical application requires further clinical validation and the development of suitable tests for routine analysis. The integromics approach provided new deeper insights into a number of mechanisms and molecular pathways affected in IVDH, which could not be observed or distinguished by exclusively monitoring the individual omics layers, not to mention current diagnostic methods. Our approach and obtained results contribute to the improvement of treatment strategies and personalized care for patients with degenerative spinal disorders.

Supplementary Materials: The following are available online at https:/ /www.mdpi.com/article/ 10.3390/ijms222111678/s1, Supplementary File S1. List of PSMs, peptides, proteins, master proteins and protein groups identified in CSF of healthy dogs and dogs with IVDH obtained by TMT-based proteomics; Supplementary File S2. Differentially abundant proteins in CSF of healthy dogs and dogs with IVDH: Supplementary File S3. Gene ontology and pathway analyses of differentially abundant proteins in CSF of healthy dogs and dogs with IVDH; Supplementary File S4. Metabolomics profiling of CSF of healthy dogs and dogs with IVDH revealing peaks, identified and annotated metabolites, metabolic pathways and metabolic maps; Supplementary File S5. Joint pathway analysis 
of differentially abundant CSF proteins and metabolites generated in MetaboAnalyst reveals the pathways affected by IVDH in dogs.

Author Contributions: Conceptualization: M.L., O.S., A.H., P.D.E., V.M.; Data curation: A.H., A.G., P.D.E.; Formal analysis A.H., A.G.; Funding acquisition: V.M., P.D.E., B.P., M.S.; Investigation: A.H., M.L., O.S., B.P., I.R., M.M., M.S.; Methodology: A.H., I.R., A.G., B.B.L., M.M.; Project administration: A.H., O.S., M.L.; Resources: V.M., P.D.E., B.P., M.S.; Software: A.G.; Supervision: A.H., M.L.; Validation: B.B.L.; Visualization: A.H., A.G., B.P.; Roles/Writing-original draft: A.H., M.L., A.G.; Writing-review \& editing: all. All authors have read and agreed to the published version of the manuscript.

Funding: This research was supported by European Commission FP7 “VetMedZg” project (Grant Number 621394) and European Regional Development Fund (Grant Agreement KK.01.1.1.04.0086). The authors declare that there is no conflict of interest.

Institutional Review Board Statement: The Ethical Committee at the Faculty of Veterinary Medicine, University of Zagreb, Croatia approved the research (No. 640-01/18-17/67; Record No. 251-6121/333-18-01).

Data Availability Statement: The mass spectrometry proteomics data have been deposited to the Consortium via the PRIDE partner repository with the dataset identifier PXD024921. Metabolomics data have been deposited to the EMBL-EBI MetaboLights database (DOI: 10.1093/nar/gkz1019, PMID:31691833) with the identifier MTBLS2689. All results are presented within the manuscript and/or Supplementary Materials.

Acknowledgments: We thank Richard Burchmore for the invaluable support in metabolomics. This research was supported by European Commission FP7 “VetMedZg" project (grant number 621394) and European Regional Development Fund (Grant Agreement KK.01.1.1.04.0086).

Conflicts of Interest: The authors declare no conflict of interest.

\section{References}

1. Bergknut, N.; Rutges, J.; Kranenburg, H.-J.; Smolders, L.; Hagman, R.; Smidt, H.-J.; Lagerstedt, A.-S.; Penning, L.; Voorhout, G.; Hazewinkel, H.; et al. The Dog as an Animal Model for Intervertebral Disc Degeneration? Spine 2011, 37, 351-358. [CrossRef]

2. Grabovac, I.; Dorner, T.E. Association between low back pain and various everyday performances. Wien. Klin. Wochenschr. 2019, 131, 541-549. [CrossRef] [PubMed]

3. Thompson, K.; Moore, S.; Tang, S.; Wiet, M.; Purmessur, D. The chondrodystrophic dog: A clinically relevant intermediate-sized animal model for the study of intervertebral disc-associated spinal pain. JOR Spine 2018, 1, e1011. [CrossRef]

4. Monchaux, M.; Forterre, S.; Spreng, D.; Karol, A.; Forterre, F.; Wuertz-Kozak, K. Inflammatory Processes Associated with Canine Intervertebral Disc Herniation. Front. Immunol. 2017, 8, 1681. [CrossRef] [PubMed]

5. Adams, M.A.; Roughley, P.J. What is intervertebral disc degeneration, and what causes it? Spine 2006, 31, 2151-2161. [CrossRef] [PubMed]

6. Evans, H.E.; Christensen, G.C. Joints and ligaments. In Miller's Anatomy of the Dog; WB Saunders Co.: Philadelphia, PA, USA, 1979; pp. 225-268.

7. Liu, C.; Yang, M.; Liu, L.; Zhang, Y.; Zhu, Q.; Huang, C.; Wang, H.; Zhang, Y.; Li, H.; Li, C.; et al. Molecular basis of degenerative spinal disorders from a proteomic perspective (Review). Mol. Med. Rep. 2020, 21, 9-19. [CrossRef] [PubMed]

8. Risbud, M.V.; Shapiro, I.M. Role of cytokines in intervertebral disc degeneration: Pain and disc content. Nat. Rev. Rheumatol. 2014, 10, 44-56. [CrossRef]

9. Vergroesen, P.-P.A.; Kingma, I.; Emanuel, K.S.; Hoogendoorn, R.J.W.; Welting, T.J.; van Royen, B.J.; van Dieën, J.H.; Smit, T.H. Mechanics and biology in intervertebral disc degeneration: A vicious circle. Osteoarthr. Cartil. 2015, 23, 1057-1070. [CrossRef]

10. Forterre, F.; Gorgas, D.; Dickomeit, M.; Jaggy, A.; Lang, J.; Spreng, D. Incidence of spinal compressive lesions in chondrodystrophic dogs with abnormal recovery after hemilaminectomy for treatment of thoracolumbar disc disease: A prospective magnetic resonance imaging study. Vet. Surg. 2010, 39, 165-172. [CrossRef] [PubMed]

11. Smith, L.J.; Nerurkar, N.L.; Choi, K.-S.; Harfe, B.D.; Elliott, D.M. Degeneration and regeneration of the intervertebral disc: Lessons from development. Dis. Model. Mech. 2011, 4, 31-41. [CrossRef]

12. Feussner, I.; Polle, A. What the transcriptome does not tell-Proteomics and metabolomics are closer to the plants' pathophenotype. Curr. Opin. Plant Biol. 2015, 26, 26-31. [CrossRef] [PubMed]

13. Baka, R.; Eckersall, D.; Horvatic, A.; Gelemanovic, A.; Mrljak, V.; McLaughlin, M.; Athanasiou, L.V.; Papaioannou, N.; Stylianaki, I.; Hanh, H.Q.; et al. Quantitative proteomics of cerebrospinal fluid using tandem mass tags in dogs with recurrent epileptic seizures. J. Proteomics 2021, 231, 103997. [CrossRef] [PubMed]

14. Willard, M.D.; Tvedten, T. Small Animal Clinical Diagnosis by Laboratory Methods, 5th ed; Elsevier Saunders: Saint Louis, MO, USA, 2004; pp. 322-331; ISBN 978-0-7216-8903-6. 
15. Khasawneh, A.H.; Garling, R.J.; Harris, C.A. Cerebrospinal fluid circulation: What do we know and how do we know it? Brain Circ. 2018, 4, 14-18. [CrossRef] [PubMed]

16. McCabe, S.D.; Lin, D.-Y.; Love, M.I. Consistency and overfitting of multi-omics methods on experimental data. Brief. Bioinform. 2020, 21, 1277-1284. [CrossRef] [PubMed]

17. Vitrinel, B.; Koh, H.W.L.; Mujgan Kar, F.; Maity, S.; Rendleman, J.; Choi, H.; Vogel, C. Exploiting Interdata Relationships in Next-generation Proteomics Analysis. Mol. Cell. Proteomics 2019, 18, S5-S14. [CrossRef] [PubMed]

18. Horvatić, A.; Guillemin, N.; Kaab, H.; McKeegan, D.; O’Reilly, E.; Bain, M.; Kuleš, J.; Eckersall, P.D. Quantitative proteomics using tandem mass tags in relation to the acute phase protein response in chicken challenged with Escherichia coli lipopolysaccharide endotoxin. J. Proteomics 2019, 192, 64-77. [CrossRef] [PubMed]

19. Lee, N.N.; Kramer, J.S.; Stoker, A.M.; Bozynski, C.C.; Cook, C.R.; Stannard, J.T.; Choma, T.J.; Cook, J.L. Canine models of spine disorders. JOR SPINE 2020, 3, e1109. [CrossRef] [PubMed]

20. Khan, A.N.; Jacobsen, H.E.; Khan, J.; Filippi, C.G.; Levine, M.; Lehman, R.A., Jr.; Riew, K.D.; Lenke, L.G.; Chahine, N.O. Inflammatory biomarkers of low back pain and disc degeneration: A review. Ann. N. Y. Acad. Sci. 2017, 1410, 68-84. [CrossRef]

21. Skouen, J.S.; Larsen, J.L.; Gjerde, I.O.; Hegrestad, S.E.; Vollset, S.E. Cerebrospinal fluid protein concentrations in patients with sciatica caused by lumbar disc herniation: An investigation of biochemical, neurologic, and radiologic predictors of long-term outcome. J. Spinal Disord. 1997, 10, 505-511. [CrossRef]

22. Stolić, I.; Škrlin, B.; Bubić Špoljar, J.; Smolec, O.; Radišić, B.; Pećin, M.; Pirkić, B.; Lipar, M. Cerebrospinal fluid assessment in dogs with spinal tumors and intervertebral disk herniation. Vet. Stanica 2020, 51, 387-392. [CrossRef]

23. Sarath Babu, N.; Krishnan, S.; Brahmendra Swamy, C.V.; Venkata Subbaiah, G.P.; Gurava Reddy, A.V.; Idris, M.M. Quantitative proteomic analysis of normal and degenerated human intervertebral disc. Spine J. 2016, 16, 989-1000. [CrossRef]

24. Erwin, W.M.; DeSouza, L.; Funabashi, M.; Kawchuk, G.; Karim, M.Z.; Kim, S.; Mädler, S.; Matta, A.; Wang, X.; Mehrkens, K.A. The biological basis of degenerative disc disease: Proteomic and biomechanical analysis of the canine intervertebral disc. Arthritis Res. Ther. 2015, 17, 240. [CrossRef] [PubMed]

25. Liu, X.-D.; Zeng, B.-F.; Xu, J.-G.; Zhu, H.-B.; Xia, Q.-C. Proteomic analysis of the cerebrospinal fluid of patients with lumbar disk herniation. Proteomics 2006, 6, 1019-1028. [CrossRef]

26. Simats, A.; Ramiro, L.; García-Berrocoso, T.; Briansó, F.; Gonzalo, R.; Martín, L.; Sabé, A.; Gill, N.; Penalba, A.; Colomé, N.; et al. A Mouse Brain-based Multi-omics Integrative Approach Reveals Potential Blood Biomarkers for Ischemic Stroke. Mol. Cell. Proteomics 2020, 19, 1921-1935. [CrossRef] [PubMed]

27. Montaner, J.; Ramiro, L.; Simats, A.; Tiedt, S.; Makris, K.; Jickling, G.C.; Debette, S.; Sanchez, J.-C.; Bustamante, A. Multilevel omics for the discovery of biomarkers and therapeutic targets for stroke. Nat. Rev. Neurol. 2020, 16, 247-264. [CrossRef] [PubMed]

28. Stoop, M.P.; Coulier, L.; Rosenling, T.; Shi, S.; Smolinska, A.M.; Buydens, L.; Ampt, K.; Stingl, C.; Dane, A.; Muilwijk, B.; et al. Quantitative Proteomics and Metabolomics Analysis of Normal Human Cerebrospinal Fluid Samples. Mol. Cell. Proteomics 2010, 9, 2063-2075. [CrossRef]

29. French, C.D.; Willoughby, R.E.; Pan, A.; Wong, S.J.; Foley, J.F.; Wheat, L.J.; Fernandez, J.; Encarnacion, R.; Ondrush, J.M.; Fatteh, N.; et al. NMR metabolomics of cerebrospinal fluid differentiates inflammatory diseases of the central nervous system. PLoS Negl. Trop. Dis. 2018, 12, e0007045. [CrossRef]

30. Sengupta, M.B.; Basu, M.; Iswarari, S.; Mukhopadhyay, K.K.; Sardar, K.P.; Acharyya, B.; Mohanty, P.K.; Mukhopadhyay, D. CSF Proteomics of Secondary Phase Spinal Cord Injury in Human Subjects: Perturbed Molecular Pathways Post Injury. PLoS ONE 2014, 9, e110885.

31. Topsakal, C.; Kilic, N.; Ozveren, F.; Akdemir, I.; Kaplan, M.; Tiftikci, M.; Gursu, F. Effects of prostaglandin E1, melatonin, and oxytetracycline on lipid peroxidation, antioxidant defense system, paraoxonase (PON1) activities, and homocysteine levels in an animal model of spinal cord injury. Spine 2003, 28, 1643-1652. [CrossRef]

32. Willkommen, D.; Lucio, M.; Moritz, F.; Forcisi, S.; Kanawati, B.; Smirnov, K.S.; Schroeter, M.; Sigaroudi, A.; Schmitt-Kopplin, P.; Michalke, B. Metabolomic investigations in cerebrospinal fluid of Parkinson's disease. PLoS ONE 2018, 13, e0208752. [CrossRef]

33. Murgia, F.; Lorefice, L.; Poddighe, S.; Fenu, G.; Secci, M.A.; Marrosu, M.G.; Cocco, E.; Atzori, L. Multi-Platform Characterization of Cerebrospinal Fluid and Serum Metabolome of Patients Affected by Relapsing-Remitting and Primary Progressive Multiple Sclerosis. J. Clin. Med. 2020, 9, 863. [CrossRef] [PubMed]

34. Sperringer, J.E.; Addington, A.; Hutson, S.M. Branched-Chain Amino Acids and Brain Metabolism. Neurochem. Res. 2017, 42, 1697-1709. [CrossRef]

35. Liu, Y.; Hyde, A.S.; Simpson, M.A.; Barycki, J.J. Emerging Regulatory Paradigms in Glutathione Metabolism. Adv. Cancer Res. 2014, 122, 69-101. [PubMed]

36. Fenves, A.Z.; Kirkpatrick, H.M.; Patel, V.V.; Sweetman, L.; Emmett, M. Increased Anion Gap Metabolic Acidosis as a Result of 5-Oxoproline (Pyroglutamic Acid): A Role for Acetaminophen. Clin. J. Am. Soc. Nephrol. 2006, 1, 441-447. [CrossRef] [PubMed]

37. Park, J.; Shin, Y.; Kim, T.H.; Kim, D.-H.; Lee, A. Plasma metabolites as possible biomarkers for diagnosis of breast cancer. PLoS ONE 2019, 14, e0225129. [CrossRef] [PubMed]

38. Hashimoto, K.; Bruno, D.; Nierenberg, J.; Marmar, C.R.; Zetterberg, H.; Blennow, K.; Pomara, N. Abnormality in glutamineglutamate cycle in the cerebrospinal fluid of cognitively intact elderly individuals with major depressive disorder: A 3-year follow-up study. Transl. Psychiatry 2016, 6, e744. [CrossRef] [PubMed] 
39. Belov Kirdajova, D.; Kriska, J.; Tureckova, J.; Anderova, M. Ischemia-Triggered Glutamate Excitotoxicity From the Perspective of Glial Cells. Front. Cell. Neurosci. 2020, 14, 51. [CrossRef]

40. Chen, Q.; Meng, Z.; Su, R. WERFE: A Gene Selection Algorithm Based on Recursive Feature Elimination and Ensemble Strategy. Front. Bioeng. Biotechnol. 2020, 8, 496. [CrossRef]

41. EL-Manzalawy, Y.; Hsieh, T.-Y.; Shivakumar, M.; Kim, D.; Honavar, V. Min-redundancy and max-relevance multi-view feature selection for predicting ovarian cancer survival using multi-omics data. BMC Med. Genom. 2018, 11, 71. [CrossRef] [PubMed]

42. Johannes, M.; Brase, J.C.; Fröhlich, H.; Gade, S.; Gehrmann, M.; Fälth, M.; Sültmann, H.; Beißbarth, T. Integration of pathway knowledge into a reweighted recursive feature elimination approach for risk stratification of cancer patients. Bioinformatics 2010, 26, 2136-2144. [CrossRef] [PubMed]

43. Duan, K.-B.; Rajapakse, J.C.; Wang, H.; Azuaje, F. Multiple SVM-RFE for gene selection in cancer classification with expression data. IEEE Trans. Nanobioscience 2005, 4, 228-234. [CrossRef] [PubMed]

44. Li, K.-C.; Wang, F.; Zhong, Y.-Q.; Lu, Y.-J.; Wang, Q.; Zhang, F.-X.; Xiao, H.-S.; Bao, L.; Zhang, X. Reduction of follistatin-like 1 in primary afferent neurons contributes to neuropathic pain hypersensitivity. Cell Res. 2011, 21, 697-699. [CrossRef] [PubMed]

45. Tulke, S.; Williams, P.; Hellysaz, A.; Ilegems, E.; Wendel, M.; Broberger, C. Nucleobindin 1 (NUCB1) is a Golgi-resident marker of neurons. Neuroscience 2016, 314, 179-188. [CrossRef] [PubMed]

46. Sy, M.; Brandt, A.U.; Lee, S.-U.; Newton, B.L.; Pawling, J.; Golzar, A.; Rahman, A.A.; Yu, Z.; Cooper, G.; Scheel, M.; et al. N-Acetylglucosamine drives myelination by triggering oligodendrocyte precursor cell differentiation. J. Biol. Chem. 2020, 295, 17413-17424. [CrossRef]

47. Yang, X.; Chen, S.; Shao, Z.; Li, Y.; Wu, H.; Li, X.; Mao, L.; Zhou, Z.; Bai, L.; Mei, X.; et al. Apolipoprotein E Deficiency Exacerbates Spinal Cord Injury in Mice: Inflammatory Response and Oxidative Stress Mediated by NF-кB Signaling Pathway. Front. Cell. Neurosci. 2018, 12, 142. [CrossRef]

48. Lind, A.-L.; Just, D.; Mikus, M.; Fredolini, C.; Ioannou, M.; Gerdle, B.; Ghafouri, B.; Bäckryd, E.; Tanum, L.; Gordh, T.; et al. CSF levels of apolipoprotein C1 and autotaxin found to associate with neuropathic pain and fibromyalgia. J. Pain Res. 2019, 12, 2875-2889. [CrossRef]

49. Salsas-Escat, R.; Stultz, C.M. The Molecular Mechanics of Collagen Degradation: Implications for Human Disease. Exp. Mech. 2009, 49, 65-77. [CrossRef]

50. Radek, M.; Pacholczyk-Sienicka, B.; Jankowski, S.; Albrecht, Ł.; Grodzka, M.; Depta, A.; Radek, A. Assessing the correlation between the degree of disc degeneration on the Pfirrmann scale and the metabolites identified in HR-MAS NMR spectroscopy. Magn. Reson. Imaging 2016, 34, 376-380. [CrossRef] [PubMed]

51. Miller, J.A.; Pappan, K.; Thompson, P.A.; Want, E.J.; Siskos, A.P.; Keun, H.C.; Wulff, J.; Hu, C.; Lang, J.E.; Chow, H.-H.S. Plasma Metabolomic Profiles of Breast Cancer Patients after Short-term Limonene Intervention. Cancer Prev. Res. 2015, 8, 86-93. [CrossRef] [PubMed]

52. Yamamoto, M.; Pinto-Sanchez, M.I.; Bercik, P.; Britz-McKibbin, P. Metabolomics reveals elevated urinary excretion of collagen degradation and epithelial cell turnover products in irritable bowel syndrome patients. Metabolomics 2019, 15, 82. [CrossRef]

53. Ågren, M.S.; Jorgensen, I.N.; Andersen, M.; Viljanto, J.; Gottrup, P. Matrix metalloproteinase 9 level predicts optimal collagen deposition during early wound repair in humans. Brit. J. Surgery 1998, 85, 68-71. [CrossRef]

54. Cao, X.; Feng, S.; Fu, C.; Gao, K.; Guo, J.; Guo, X.; He, X.; Huang, Z.; Li, Z.; Liu, L.; et al. Repair, protection and regeneration of spinal cord injury. Neural Regen. Res. 2015, 10, 1953. [CrossRef] [PubMed]

55. Albayar, A.A.; Roche, A.; Swiatkowski, P.; Antar, S.; Ouda, N.; Emara, E.; Smith, D.H.; Ozturk, A.K.; Awad, B.I. Biomarkers in Spinal Cord Injury: Prognostic Insights and Future Potentials. Front. Neurol. 2019, 10, 27. [CrossRef] [PubMed]

56. Wu, Y.; Streijger, F.; Wang, Y.; Lin, G.; Christie, S.; Mac-Thiong, J.-M.; Parent, S.; Bailey, C.S.; Paquette, S.; Boyd, M.C.; et al. Parallel Metabolomic Profiling of Cerebrospinal Fluid and Serum for Identifying Biomarkers of Injury Severity after Acute Human Spinal Cord Injury. Sci. Rep. 2016, 6, 38718. [CrossRef] [PubMed]

57. Mangaraj, M.; Nanda, R.; Panda, S. Apolipoprotein A-I: A Molecule of Diverse Function. Indian J. Clin. Biochem. 2016, 31, 253-259. [CrossRef] [PubMed]

58. Lepennetier, G.; Hracsko, Z.; Unger, M.; van Griensven, M.; Grummel, V.; Krumbholz, M.; Berthele, A.; Hemmer, B.; Kowarik, M.C. Cytokine and immune cell profiling in the cerebrospinal fluid of patients with neuro-inflammatory diseases. J. Neuroinflamm. 2019, 16, 219. [CrossRef]

59. Simats, A.; García-Berrocoso, T.; Penalba, A.; Giralt, D.; Llovera, G.; Jiang, Y.; Ramiro, L.; Bustamante, A.; Martinez-Saez, E.; Canals, F.; et al. CCL 23: A new CC chemokine involved in human brain damage. J. Intern. Med. 2018, 283, 461-475. [CrossRef]

60. Rabin, R.L. CC, C, and CX3C Chemokines, Encyclopedia of Hormones; Academic Press: Cambridge, MA, USA, 2003; pp. $255-263$. [CrossRef]

61. Könnecke, H.; Bechmann, I. The Role of Microglia and Matrix Metalloproteinases Involvement in Neuroinflammation and Gliomas. Clin. Dev. Immunol. 2013, 2013, 1-15. [CrossRef] [PubMed]

62. Mlekusch, R.; Humpel, C. Matrix metalloproteinases-2 and -3 are reduced in cerebrospinal fluid with low beta-amyloid1-42 levels. Neurosci. Lett. 2009, 466, 135-138. [CrossRef] [PubMed]

63. Chen, L.; Deng, H.; Cui, H.; Fang, J.; Zuo, Z.; Deng, J.; Li, Y.; Wang, X.; Zhao, L. Inflammatory responses and inflammationassociated diseases in organs. Oncotarget 2018, 9, 7204-7218. [CrossRef] [PubMed] 
64. Magdalon, J.; Mansur, F.; Teles e Silva, A.L.; de Goes, V.A.; Reiner, O.; Sertié, A.L. Complement System in Brain Architecture and Neurodevelopmental Disorders. Front. Neurosci. 2020, 14, 23. [CrossRef] [PubMed]

65. Gompels, M.M.; Lock, R.J.; Abinun, M.; Bethune, C.A.; Davies, G.; Grattan, C.; Fay, A.C.; Longhurst, H.J.; Morrison, L.; Price, A.; et al. C1 inhibitor deficiency: Consensus document. Clin. Exp. Immunol. 2005, 139, 379-394. [CrossRef]

66. Gerdle, B.; Ghafouri, B. Proteomic studies of common chronic pain conditions-A systematic review and associated network analyses. Expert Rev. Proteom. 2020, 17, 483-505. [CrossRef]

67. Teckchandani, S.; Nagana Gowda, G.A.; Raftery, D.; Curatolo, M. Metabolomics in chronic pain research. Eur. J. Pain 2021, 25, 313-326. [CrossRef] [PubMed]

68. Pfyffer, D.; Wyss, P.O.; Huber, E.; Curt, A.; Henning, A.; Freund, P. Metabolites of neuroinflammation relate to neuropathic pain after spinal cord injury. Neurology 2020, 95, e805-e814. [CrossRef] [PubMed]

69. Jung, C.; Ichesco, E.; Ratai, E.-M.; Gonzalez, R.G.; Burdo, T.; Loggia, M.L.; Harris, R.E.; Napadow, V. Magnetic resonance imaging of neuroinflammation in chronic pain: A role for astrogliosis? Pain 2020, 161, 1555-1564. [CrossRef] [PubMed]

70. Zhou, C.; Simpson, K.L.; Lancashire, L.J.; Walker, M.J.; Dawson, M.J.; Unwin, R.D.; Rembielak, A.; Price, P.; West, C.; Dive, C.; et al. Statistical Considerations of Optimal Study Design for Human Plasma Proteomics and Biomarker Discovery. J. Proteome Res. 2012, 11, 2103-2113. [CrossRef]

71. Bharucha, T.; Gangadharan, B.; Kumar, A.; de Lamballerie, X.; Newton, P.N.; Winterberg, M.; Dubot-Pérès, A.; Zitzmann, N. Mass spectrometry-based proteomic techniques to identify cerebrospinal fluid biomarkers for diagnosing suspected central nervous system infections. A systematic review. J. Infect. 2019, 79, 407-418. [CrossRef] [PubMed]

72. Bednarski, R.; Grimm, K.; Harvey, R.; Lukasik, V.M.; Penn, W.S.; Sargent, B.; Spelts, K. American Animal Hospital Association. AAHA anesthesia guidelines for dogs and cats. J. Am. Anim. Hosp. Assoc. 2011, 7, 377-385. [CrossRef] [PubMed]

73. Perez-Riverol, Y.; Csordas, A.; Bai, J.; Bernal-Llinares, M.; Hewapathirana, S.; Kundu, D.J.; Inuganti, A.; Griss, J.; Mayer, G.; Eisenacher, M.; et al. The PRIDE database and related tools and resources in 2019: Improving support for quantification data. Nucleic Acids Res. 2019, 47, D442-D450. [CrossRef]

74. Tvarijonaviciute, A.; Tecles, F.; Caldin, M.; Tasca, S.; Cerón, J. Validation of spectrophotometric assays for serum paraoxonase type-1 measurement in dogs. Am. J. Vet. Res. 2012, 73, 34-41. [CrossRef]

75. Weidmeyer, C.E.; Solter, P.F. Validation of human haptoglobin immunoturbidimetric assay for detection of haptoglobin in equine and canine serum and plasma. Vet. Clin. Pathol. 1996, 25, 141-146. [CrossRef] [PubMed]

76. Thomas, F.C.; Mudaliar, M.; Tassi, R.; McNeilly, T.N.; Burchmore, R.; Burgess, K.; Herzyk, P.; Zadoks, R.N.; Eckersall, P.D. Mastitomics, the integrated omics of bovine milk in an experimental model of Streptococcus uberis mastitis: 3 . Untargeted metabolomics. Mol. Biosyst. 2016, 12, 2762-2769. [CrossRef]

77. Haug, K.; Cochrane, K.; Nainala, V.C.; Williams, M.; Chang, J.; Jayaseelan, K.V.; O'Donovan, C. MetaboLights: A resource evolving in response to the needs of its scientific community. Nucleic Acids Res. 2019, 48, D440-D444. [CrossRef] [PubMed]

78. Gloaguen, Y.; Morton, F.; Daly, R.; Gurden, R.; Rogers, S.; Wandy, J.; Wilson, D.; Barrett, M.; Burgess, K. PiMP my metabolome: An integrated, web-based tool for LC-MS metabolomics data. Bioinformatics 2017, 33, 4007-4009. [CrossRef] [PubMed]

79. Holman, J.D.; Tabb, D.L.; Mallick, P. Employing ProteoWizard to Convert Raw Mass Spectrometry Data. Curr. Protoc. Bioinforma. 2014, 46, 13-24. [CrossRef] [PubMed]

80. R Core Team. R: A Language and Environment for Statistical Computing; R Core Team: Vienna, Austria, 2020.

81. Komsta, L. Outliers: Tests for outliers. In R Package Version 0.14; R Core Team: Vienna, Austria, 2011.

82. Wickham, H. About the ggplot2 Package. J. Appl. Comput. Math. 2016, 5, 4. [CrossRef]

83. Szklarczyk, D.; Gable, A.L.; Lyon, D.; Junge, A.; Wyder, S.; Huerta-Cepas, J.; Simonovic, M.; Doncheva, N.T.; Morris, J.H.; Bork, P.; et al. STRING v11: Protein-protein association networks with increased coverage, supporting functional discovery in genome-wide experimental datasets. Nucleic Acids Res. 2019, 47, D607-D613. [CrossRef] [PubMed]

84. Shannon, P. Cytoscape: A Software Environment for Integrated Models of Biomolecular Interaction Networks. Genome Res. 2003, 13, 2498-2504. [CrossRef]

85. Huang, D.W.; Sherman, B.T.; Lempicki, R.A. Systematic and integrative analysis of large gene lists using DAVID bioinformatics resources. Nat. Protoc. 2009, 4, 44-57. [CrossRef]

86. Kuhn, M. Classification and Regression Training. R Package Version 6.0-86. 2020. Available online: https://cran.r-project.org/ web/packages/caret/index.html (accessed on 14 May 2021).

87. Friedman, J.; Hastie, T.; Tibshirani, R. Regularization Paths for Generalized Linear Models via Coordinate Descent. J. Stat. Softw. 2010, 33, 1-22. [CrossRef] [PubMed]

88. De Jay, N.; Papillon-Cavanagh, S.; Olsen, C.; El-Hachem, N.; Bontempi, G.; Haibe-Kains, B. mRMRe: An R package for parallelized mRMR ensemble feature selection. Bioinformatics 2013, 29, 2365-2368. [CrossRef] [PubMed]

89. Zhou, Y.; Zhou, B.; Pache, L.; Chang, M.; Khodabakhshi, A.H.; Tanaseichuk, O.; Benner, C.; Chanda, S.K. Metascape provides a biologist-oriented resource for the analysis of systems-level datasets. Nat. Commun. 2019, 10, 1523. [CrossRef] [PubMed] 